\title{
Modelling Shared Space Users Via Rule-based Social Force Model
}

\author{
Bani Anvari ${ }^{\mathrm{a}}$, Michael G.H. Bell, ${ }^{\mathrm{a}, \mathrm{b}}$, Aruna Sivakumar ${ }^{\mathrm{a}}$, Washington Y. Ochieng ${ }^{\mathrm{a}}$ \\ ${ }^{a}$ Centre for Transport Studies, Department of Civil and Environmental Engineering, Imperial College \\ London, SW7 2BU, UK \\ ${ }^{b}$ Institute of Transport and Logistics Studies, University of Sydney Business School, NSW
}

\begin{abstract}
The promotion of space sharing in order to raise the quality of community living and safety of street surroundings is increasingly accepted feature of modern urban design. In this context, the development of a shared space simulation tool is essential in helping determine whether particular shared space schemes are suitable alternatives to traditional street layouts. A simulation tool that enables urban designers to visualise pedestrians and cars trajectories, extract flow and density relation in a new shared space design, achieve solutions for optimal design features before implementation, and help getting the design closer to the system optimal. This paper presents a three-layered microscopic mathematical model which is capable of representing the behaviour of pedestrians and vehicles in shared space layouts and it is implemented in a traffic simulation tool. The top layer calculates route maps based on static obstacles in the environment. It plans the shortest path towards agents' respective destinations by generating one or more intermediate targets. In the second layer, the Social Force Model (SFM) is modified and extended for mixed traffic to produce feasible trajectories. Since car movements are not as flexible as pedestrian movements, velocity angle constraints are included for cars. The conflicts described in the third layer are resolved by rule-based constraints for shared space users. An optimisation algorithm is applied to determine the interaction parameters of the force-based model for shared space users using empirical data. This new three-layer microscopic model can be used to simulate shared space environments and assess, for example, new street designs.
\end{abstract}

\section{Keywords}

Shared space; Social Force Model; Flood fill algorithm; Mixed traffic; Mathematical microscopic model 


\section{Shared Space Background}

Since the appearance of motorised transport, there have been discussions about the extent to which standardised and vehicle-dominated streetscapes have had a negative effect on the environment of public neighbourhoods. Hamilton-Bailie (2008) explains that individuals will tend to spend less time in public areas, if they perceive streets to be less attractive for their social interaction activities or transport movements. As a result, the quality of these spaces will decline and human activities will be transferred from public to private spaces. Hence, urban design is promoting shared space as an alternative to traditional designs (Hamilton-Baillie, 2008; Gaffikin, et al., 2010; Dumbaugh \& Li, 2011; Department for Communities and Local Government-UK, 2012; Schonauer, et al., 2012).

Space sharing (see Table 1) has been initiated by the woonerf (living playground) concept in the Netherlands in the late 1960s. A woonerf is a residential street, designed to provide safe and pleasurable areas for pedestrians (specifically children), and where pedestrians are given priority over motor vehicles. In particular, a woonerf street is designed without a clear segregation between pedestrians and cars, so that all road users should travel with caution. In the UK, from the late 1990s, a woonerf was known as a home zone. In the 1980s, some of the ideas and characteristics of the woonerf approach were introduced to traffic calming design. Compared to woonerfs, traffic calming schemes are focused only on slowing down traffic and they lack elements to improve social interactions. In addition, vehicular traffic is given priority over pedestrians.

Table 1

The characteristics of new design approaches (Shearer, 2010)

\begin{tabular}{|c|c|c|c|}
\hline & Woonerf & Traffic Calming & Shared Space \\
\hline Alternative Names & $\begin{array}{l}\text { Home Zone } \\
\text { Residential Yard }\end{array}$ & Traffic Control & $\begin{array}{l}\text { Naked Intersection } \\
\text { Liveable Streets } \\
\text { Shared Streets/Zones }\end{array}$ \\
\hline Land Use & Residential & Any Land Use & Any Land Use \\
\hline $\begin{array}{l}\text { Is Social Interaction an } \\
\text { Aim? }\end{array}$ & Yes & No & No \\
\hline Design Approach & Flexible & Standardised & Flexible \\
\hline Objective & $\begin{array}{l}\text { Slow Traffic to Allow } \\
\text { Social Interaction }\end{array}$ & Slow Traffic & Multi-faceted \\
\hline Who has the Priority? & Pedestrians & Traffic & Identical \\
\hline Initiated in & $1960 \mathrm{~s}$ & $1980 \mathrm{~s}$ & 1991 (first applied in 2004) \\
\hline
\end{tabular}


The most recent version of the space sharing idea is called "shared space". This is a concept that promotes a sense of vigilance and responsibility by reducing demarcations and any physical distinction between the streets and pedestrian areas. Attractive features are added to shared space environments to provide a pleasurable area that stimulate pedestrians to walk to their destinations. While woonerf and home zones are small scale examples of doing away with the traditional separation between the footpath and carriageway, the shared space approach is flexible in use of street space and the right-of-way assigned to road users (see Table 1). Shared space is "an approach to improving streets and places where both pedestrians and vehicles are present, with layouts related more to the pedestrian scale and with features encouraging

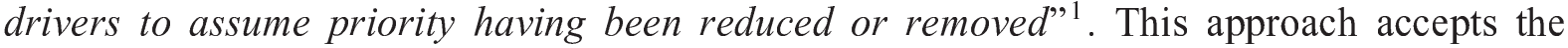
important function of the road as a transport link for the surrounding area, while creating an integrated street environment for pedestrians and vehicles through the use of a single surface pavement, with minimal traffic signals and road markings (Department for Transport of UK, 2011).

Debate on the development of shared space environments is largely polarised between those who view shared space as essential to the improvement of social lifestyle quality and safety, and those sceptical about its adoption due to a lack of research on shared space perceptions and on the safety of road users.

Gaventa (2004) identified shared spaces as slow and safe environments that develop social negotiation between pedestrians and drivers with the aim of providing civilised urban places. Hamilton-Baillie (2008) argued that the success of space sharing depends on changing the road users' understanding of safety and risk within a mixed environment. New designs should be structured in a way that encourages behavioural changes among users. Monderman (2007)stated that the absence of demarcations and traffic facilities in shared spaces provokes uncertainties in both drivers and pedestrians and this can results in slower driving speeds and an increased safety awareness. In this environment, road users will become more conscious of the movement patterns around them by perceiving interactions via eye contact and by predicting behaviours. Ben-Joseph (1995) and Hamilton-Baillie (2008) argued that shared space can increase the safety of street spaces and consequently decrease the amount of fatal accidents and injuries. Southworth and Ben-Joseph (2003) sought to prove this idea based on observations and research reports in different countries where shared spaces have been applied. They concluded that shared space contributes to fewer vehicle movements than traditional traffic separation.

Quimby and Castle (2006), however, criticised the results reported by Southworth and Ben-Joseph (2003) arguing that they were based on small, isolated locations with low traffic flows. They added that the cultural and social context of the studied areas were critical elements that could significantly impact the results in different countries. Melia (2012) believes that separation is required when the aim is to encourage a specific traffic mode, protect nonmotorised modes and give a feeling of safety to non-motorised users in cities and towns. $\mathrm{He}$ added that shared space schemes can contribute to these aims, particularly when the traffic volumes are low. Jenks (1983) and Clayden et al. (2006) argued against shared space by noting that some road users would neither accept nor understand the allocation of rights, with the result that space sharing is unsafe for walking. Moreover, the Guide Dogs for the Blind Association (GDB) (2007) in London voiced concerns about vision impaired people, who are usually dependent on kerb lines and spatial settings. It recommended that this feature should be considered when implementing shared space. Given the concerns above, Quimby and Castle

\footnotetext{
${ }^{1}$ Kaparias, I. et al., 2012. Analysing the perceptions of pedestrians and drivers to shared space. Transportation Research Part F: Traffic Psychology and Behaviour, 15(3), pp. 297-310.
} 
(2006) argued that the implementation of the shared space concept needs to be adapted to fit the big cities and town centres' environment. Luca et al. (2012) supported this idea, emphasizing the importance of studying the social, environmental, economical and behavioural factors of a given city before implementing a shared space environment in city centres.

Shared space can be considered as a design alternative to traditional traffic separation taking into account traffic volumes, land uses, vulnerable users and size of the potential area (Gerlach \& Methorst, 2009). As with all design plans, shared space struggles to achieve all its theoretical objectives dependant on different contexts (Gerlach \& Methorst, 2009). The visualisation, evaluation and environmental response of pedestrians and drivers are important criteria in achieving a successful, socially connected shared space (Sutcliffe, 2009). These criteria depend on the way new designs influence the perception of road users (Hamilton-Baillie, 2008), as well as on social culture in terms of the understanding and acceptance of shared space as a safe scheme (Quimby \& Castle, 2006).

\section{Research Problem and Contribution}

A number of shared space schemes have already been implemented around the world without prior evaluations (see Table 2). There is a need to explore optimal shared space design options prior to real implementation in order to evaluate their efficiency (average road user delays and road capacity), safety (initial time-to-collision) and environmental impacts (emissions based on instantaneous speed and acceleration of vehicles). Modelling and simulation in combination with visualisation of pedestrian and vehicle trajectories in future shared space designs will confirm or disprove the potential outcomes of these schemes. Hence, urban designers can for instance visualise the trajectories of pedestrian and cars, extract flow and density relationship in the design stage of new shared space areas and identify the conditions under which shared space might be a feasible alternative to traditionally controlled traffic designs.

Table 2

A number of shared space schemes worldwide (Quimby \& Castle, 2006; Shearer, 2010)

\begin{tabular}{ll}
\hline Country & Towns/Councils \\
\hline Australia & Bendigo \\
\hline Austria & Feldkirchen bei Graz, Gleinstätten, Graz, Vöcklabruck, Gnas, Velden am Wörther See \\
\hline Belgium & Ostende \\
\hline Denmark & Ejby \\
\hline Germany & Blomberg, Bohmte, Calau, Duisburg, Endingen am Kaiserstuhl, Ettenheim, Frankfurt am \\
& Main, Hamburg, Haslach im Kinzigtal, Luckenwalde, Potsdam, Rudersberg, Wolfach, \\
& Hatten OT Kirchhatten \\
\hline Netherlands & Emmen, Donkerbroek, Drachten, Makkinga, Nijega, Oldeberkoop, \\
& Opeinde, Oosterwolde, Olderberkoop, Oudehaske, Wolvega, Haren \\
\hline New Zealand & Auckland, Christchurch, Dunedin, Hamilton, Napier, Nelson, Orewa, \\
& Papakura, Waitakera, Wellington \\
\hline Sweden & Norrköping \\
\hline United Kingdom & Ashford, Bath, Brighton, Hove, Caernarfon, Leeds, London, Newbury, \\
& Newcastle, Oxford, Shrewsbury, Southampton, Taunton, Woking \\
\hline United States & West Palm Beach \\
\hline
\end{tabular}

Many theoretical models have been proposed in the literature to uncover the laws which govern vehicle and pedestrian traffic dynamics separately (Chraibi, et al., 2010; Franca, et al., 2009; Zhang \& Kim, 2005). However, the complex interactions of pedestrians and drivers in shared space environments cannot be mathematically described by available well-known 
software such as VISSIM or Aimsum (Alexandersson and Johansson, 2013). To the best knowledge of the authors, there is currently no simulation model which appropriately applies the shared space philosophy of equal priority and single surface modelling (Alexandersson \& Johansson, 2013). Hence, there is a need for a unified theory to explain both vehicle and pedestrian movements in shared space environments utilising a full consideration of the shared space philosophy of identical priority and single surface modelling.

This paper develops a new three-layered mathematical model for heterogeneous agents (vehicles and pedestrians) in a shared space environment with single surface pavements, no lane discipline and identical priority for all road users. The model presented here focuses on the implementation of human behaviour as pedestrians and car drivers in ideal shared space schemes without considering safety aspects. The state-of-the-art in pedestrian-vehicular mixed traffic modelling is explained in Section 3.

\section{Pedestrian-Vehicular Mixed Traffic Modelling}

Rule-based models (Jiang \& Wu, 2006; Zhang \& Chang, 2010; Zhang \& Chang, 2011; Ottomanelli, et al., 2012; Li, et al., 2012), such as Cellular Automata (CA), and force-based models (Helbing, et al., 2005; Ishaque \& Noland, 2007; Bonisch \& Kretz, 2009; Pretto, et al., 2011; Schonauer, et al., 2012; Suh, et al., 2013; Waizman, et al., 2012), such as the Social Force Model (SFM) are two of the most widely used microscopic models for pedestrian-vehicular mixed traffic.

In CA models, the intersection space is divided into discrete cells and road users follow a set of predefined rules for their local motion. Jiang and Wu (2006) and Jian et al. (2011) explored a lattice gas model for vehicle and pedestrian flows and presented vehicles' changes in speed along a narrow channel for avoiding collision with pedestrians. Zhang and Chang (2011), meanwhile, investigated the use of the CA model for simulating vehicle-pedestrian interactions. A conflict ("competition") is detected when a cell is assigned as the target for multiple agents. In this case, the waiting time of an individual is considered as a factor in winning the "competition". Ottomanelli et al. (2012) also used the CA model to simulate interactions at a road crossing. They derive the parameters from a probabilistic distribution to consider the heterogeneity of the pedestrians. CA models make simulation of mixed traffic computationally efficient, since a rule set is applied over many time steps rather than finding solutions for differential equations. On the other hand, CA models are "discrete in nature" (Jasti \& Higgs, 2005) and they may not efficiently explain the cause of an unexpected macroscopic behaviour when it emerges from the locally defined interaction.

Social force-based models are based on Newton's second law of dynamics. These models reproduce successfully a large set of collective phenomena involving pedestrians such as lane formation (Helbing \& Molnar, 1995; Yu, et al., 2005), oscillations at bottle-necks (Helbing \& Molnar, 1995), faster-is-slower effects (Parisi \& Dorso, 2007; Helbing, et al., 2000; Lakoba, et al., 2005) and clogging at exit doors (Yu, et al., 2005). Different force-based models have mathematically formulated a more realistic behaviour by modifying the SFM by Helbing and Molnar (1995). The parameters used in these models can be interpreted and their range of values can be measured by comparing real data (Lakoba, et al., 2005). The SFM can be reduced to one dimension and describe car following behaviour. In addition, it assigns identical priority to all users and provides constant agent movements on a single surface. As a result, force-based models offer the possibility of a unified theory that can explain both vehicle and pedestrian movements, both separately and in interaction with each other. The set of parameters' changes based on the implementation of the model and the simulated geometry. Furthermore, the implementations of social force-based models often require additional elements to ensure 
adequate realism. For instance, overlapping of agents or negative velocities sometimes occur. These issues need to be resolved by overriding the equation of motion.

Of the various force-based models Helbing et al. (2005) formulated and analysed the interaction of pedestrians with vehicles in crossing sections. Pedestrian traffic was examined by Ishaque and by Noland (2007) and Bonisch and Kretz (2009) using VISSIM where vehicle and pedestrian modes operate independently and are controlled by traffic signals at conflict areas. Pretto et al. (2011) used a combination of force directed and rule-based approaches for modelling interactions of pedestrians and vehicles at crossing points. The interaction between pedestrians and vehicles in shared space environments is modelled for pedestrian behaviours using the SFM in Schonauer et al. (2012). In this model, a single track approach is used for vehicle dynamics and a game theoretic approach is assigned for resolving conflicts between pedestrians and vehicles in shared space scenarios. This model is very accurate but computationally expensive. Suh et al. (2013) utilised the SFM implemented in VISSIM for capturing gap-seeking behaviour of pedestrians at a crosswalk with priority rules.

The aim of this paper is to simulate pedestrians and 4-wheeled motorised vehicles in shared space areas based on a force-based mathematical framework. The suitable and essential characteristics of pedestrians and drivers in shared space schemes are classified in Section 4. The analysis of elementary behaviour patterns results in a structure for the conceptual framework which consists of three interrelated layers (see Section 4). The variables used in the

formulations of the shared space model are listed in Section 1.2 and the geometrical model of four-wheeled agents is described in Section 4.2.

\section{Shared Space Behaviours and Conceptual Framework}

After investigating several real scenarios from the shared space scheme at New Road in Brighton and the analyses in Hamilton-Bailie (2008) and Schonauer (2012), a number of salient characteristics and traffic behaviours are considered for mathematical modelling:

- Shared space users should follow the shortest path to their destination according to the static obstacles and infrastructure of the environment. Obstacles should be defined as a guiding effect on the traffic behaviour of shared space users.

- The way pedestrians use space in shared space areas should be modelled by considering design characteristic (e.g. a single surface) that serve to reduce priority for car drivers and to increase priority for pedestrians. Encouraging all road users to share the available road space and balancing priority is a major objective of the shared space concept.

- Shared space should be modelled in its totality with no lane discipline while in order to reduce traffic speed, road users should be modelled dynamically by adapting to each other's behavioural changes. The desired speed as well as the maximum speed should be defined. Traffic calming and reducing stop-and-go behaviour is another objective of the shared space concept.

It is worth noting that the stated list of behaviours is not necessarily sufficient to model all shared space schemes, however, it could be used as the generic simulation framework for refining future projects. Shared spaces are designed to encourage motorised and non-motorised users to move freely in a two dimensional space without lanes for vehicles. It is assumed that road users are consequently more conscious of their surroundings and thus actively seek to avoid conflicts. This behaviour should be modelled by considering the traffic factors involved in encounters, such as: road users' speed, direction of movement, minimum distance from each other, the surrounding information and the movement constraints. 
Building on the shared space concept, the key characteristics of pedestrians and vehicles are integrated in a framework of three interrelated layers (similar to the framework of pedestrian dynamic theories) for modelling the motion of pedestrians and cars: the trajectory planning layer, force-based modelling layer and rule-based layer (see Fig. 1).

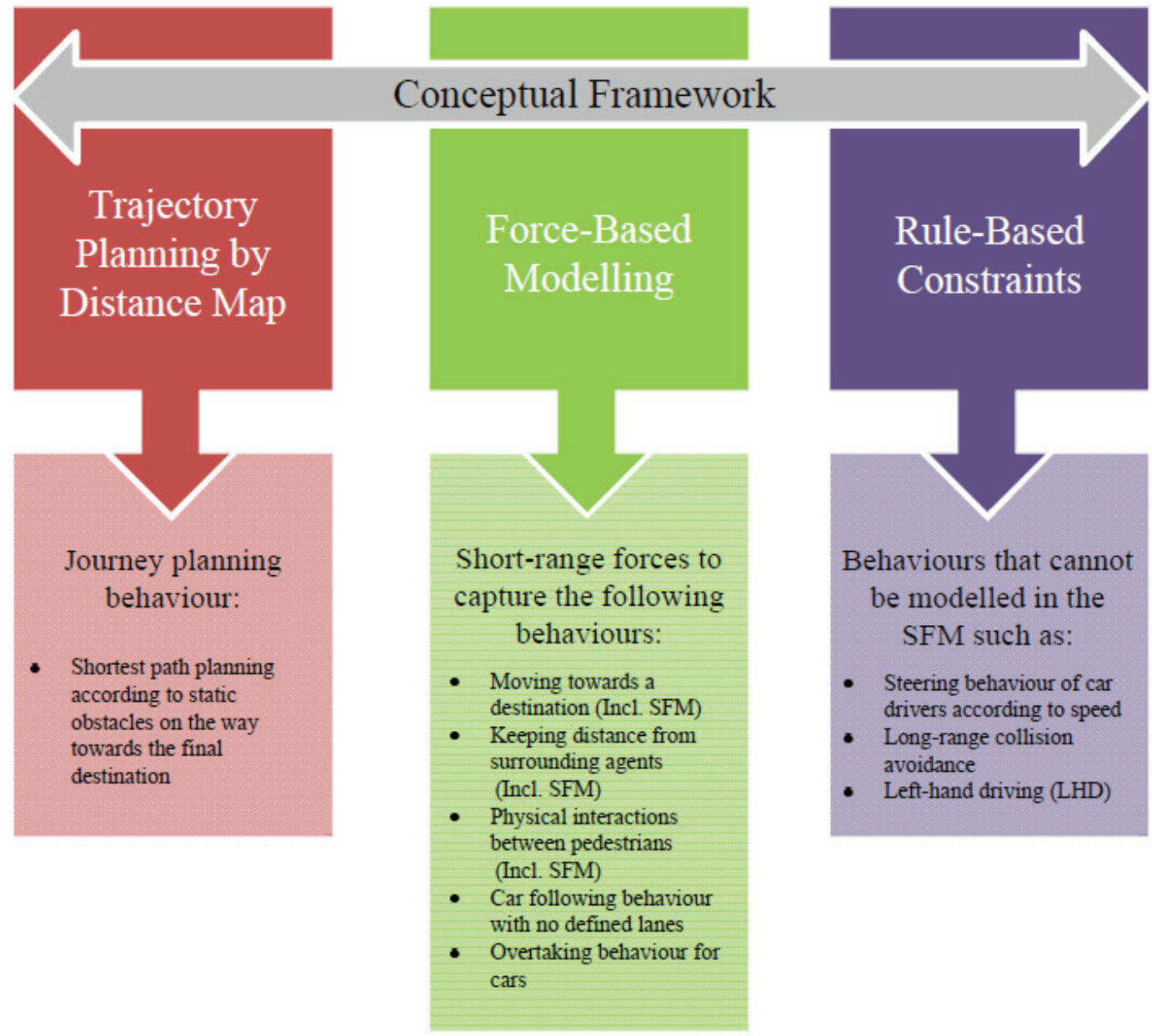

Fig. 1. Conceptual framework for capturing identical priority on single surface shared streets

The first layer, the trajectory planning layer, minimises unnecessary detours by pedestrians and cars by calculating the shortest path to the final destination via intermediate destinations with respect to the infrastructure of the shared area (see Section 5). The second layer, the forcebased layer, uses the SFM and modifies it to generate individual trajectories of pedestrians and cars from their starting point following the shortest path. Road users are assigned social/physical forces in this layer in order to reproduce their interaction, their negotiations regarding the right of way and their obstacle avoidance behaviour. In addition, the overtaking and car following behaviours of car drivers with no defined lanes are captured in this layer (see Section 0 ). In the third layer, the rule-based layer, the motion limitation of cars is taken into account by means of a relationship between the steering angle and speed considering the centrifugal acceleration expressed by the driver. In case of potential encounters, rules are assigned to avoid collision. Conflict handling manoeuvres are described and resolved with conflict avoidance constraints in Section 7. By using the conflict avoidance strategy, left-hand traffic is introduced for car-car interactions when passing in opposite directions. In order to calibrate the interaction parameters in the social force-based layer, a procedure is adapted in Section 0 to fit the quantitative data of New Road (Brighton). Section 10 summarises and concludes the main research contributions. 


\begin{tabular}{|c|c|c|c|}
\hline Variables & Explanation & Variables & Explanation \\
\hline$A$ & Interaction strength & $\boldsymbol{f}^{\text {conflict }}$ & Conflict avoidance force \\
\hline$a$ & Acceleration & $f^{\text {following }}$ & Car-following force \\
\hline$a^{\text {Centrifugal }}$ & Centrifugal acceleration & $\boldsymbol{f}^{p h, \text { friction }}$ & Friction physical force \\
\hline$B$ & Interaction range & $f^{\text {norm }}$ & Pushing physical force \\
\hline$B^{\prime}$ & Acceleration interaction range & $\boldsymbol{f}^{p h}$ & Physical force \\
\hline$B^{\prime \prime}$ & Braking interaction range & $f^{s o c}$ & Social force \\
\hline c & Cost function & $f^{\tan }$ & Tangential force \\
\hline$d$ & $\begin{array}{l}\text { Distance between the centre of two } \\
\text { shared space users }\end{array}$ & $\boldsymbol{f}^{0}$ & Driving force \\
\hline$d_{c}$ & Safe distance & $2 l$ & Length of a car \\
\hline$d_{\gamma}$ & Minimal distance of a car & $L$ & $\begin{array}{l}\text { Distance between the front and the rear } \\
\text { axle }\end{array}$ \\
\hline$d\left(v_{\gamma \delta}\right)$ & $\begin{array}{lll}\text { Speed-dependent } & \text { safe } & \text { distance } \\
\text { between two cars } & & \\
\end{array}$ & $\lambda$ & Form factor constant \\
\hline$d^{\mathrm{CPA}}$ & $\begin{array}{l}\text { Minimum distance between the agents } \\
\text { at their Closest Point of Approach } \\
\text { (CPA) }\end{array}$ & $\boldsymbol{n}$ & Normalised vector \\
\hline$d$ & Desired destination vector & $\varphi$ & $\begin{array}{l}\text { Angle between the desired direction } \\
\text { and centre of another agent }\end{array}$ \\
\hline$D^{\mathrm{C}}$ & $\begin{array}{l}\text { Chessboard distance between two } \\
\text { points }\end{array}$ & $\psi$ & Steering angle \\
\hline$D^{\text {Euclidean }}$ & Euclidean distance between two points & $q$ & Effective factor \\
\hline$D^{\mathrm{M}}$ & $\begin{array}{l}\text { Manhattan distance between two } \\
\text { points }\end{array}$ & $r$ & Radius \\
\hline$D^{\mathrm{V} 2}$ & Variant 2 distance between two points & $r_{\alpha}$ & Radius of a pedestrian \\
\hline$\delta x_{i}$ & Horizontal distance between two point & $r_{\alpha U}$ & $\begin{array}{l}\text { Sum of the radii of a pedestrian } \alpha \text { and } \\
\text { another agent } U\end{array}$ \\
\hline$\delta y_{i}$ & Vertical distance between two points & $s$ & Clearance \\
\hline$\Delta x$ & $\begin{array}{l}\text { Horizontal distance between two } \\
\text { agents }\end{array}$ & $t$ & Time \\
\hline$\Delta y$ & Vertical distance between two agents & $\tau$ & Relaxation time or reaction time \\
\hline$\Delta v_{\gamma \delta}$ & Velocity difference of two cars & $\tau_{\gamma}^{\prime}$ & Braking time of car $\gamma$ \\
\hline$v^{\min }$ & Minimum velocity change & $\Theta$ & Function depending on its argument \\
\hline$\xi$ & Fluctuation force & $T_{\gamma}$ & Safe time headway \\
\hline$e^{0}$ & Desired direction & $U$ & Shared space user (pedestrian or car) \\
\hline$F$ & Form factor & $\mathrm{v}^{0}$ & Desired speed \\
\hline $\boldsymbol{f}_{\gamma(\gamma-1)}^{\text {repulsive }}$ & $\begin{array}{l}\text { Repulsive force between car } \gamma \text { and the } \\
\text { car ahead }(\gamma-1)\end{array}$ & $v^{\text {opt }}$ & Optimal speed \\
\hline $\boldsymbol{f}_{\alpha \beta}$ & $\begin{array}{l}\text { Interaction/Repulsive force between } \\
\text { pedestrian } \alpha \text { and pedestrian } \beta\end{array}$ & $\boldsymbol{v}$ & Actual velocity \\
\hline $\boldsymbol{f}_{\alpha b}$ & $\begin{array}{l}\text { Interaction/Repulsive force between } \\
\text { pedestrian } \alpha \text { and boundary } b\end{array}$ & $\tau_{\alpha}^{k}$ & Intermediate destination \\
\hline $\boldsymbol{f}_{\alpha \delta}$ & $\begin{array}{l}\text { Interaction/Repulsive force between } \\
\text { pedestrian } \alpha \text { and car } \delta\end{array}$ & $\Delta v$ & Relative velocity \\
\hline $\boldsymbol{f}_{\delta \gamma}$ & $\begin{array}{l}\text { Interaction/Repulsive force between } \\
\text { two cars }\end{array}$ & $2 w$ & Width of a car \\
\hline
\end{tabular}

\subsection{Geometrical Agent Modelling}

In the SFM for pedestrians developed by Helbing, Farkas and Vicsek (2000), each simulated pedestrian has a certain body size which can be expressed by circles (symmetrical 
configuration) of a radius $r_{\alpha}$. This is translated to the average area occupied by a pedestrian. Since the definition of shared space is the integration of pedestrians and vehicles, a car is now introduced by an ellipse with the radius $r_{\gamma}\left(\varphi_{\gamma U}\right)$. As shown in Fig. 2, the radius $r_{\gamma}\left(\varphi_{\gamma U}\right)$ depends on the angle between the desired direction of a car and the direction of a close-by pedestrian $U=\alpha$ or car $U=\delta$. The radius of the ellipse $r_{\gamma}\left(\varphi_{\gamma U}\right)$ in polar coordinates is described by Eq. (1). Here, $2 l$ and $2 w$ are the average length and width of a modelled car and the relative distance between a car and another agent varies based on their relative angle $\varphi_{\gamma U}$. For instance, if the agents are collinear, the relative distance is maximised and when they are perpendicular, this value is minimised. At any relative angle, the distance lies between the minimum and maximum.

Considering the pedestrian as a circle and the car as an ellipse, the following sections present a model which can be used to describe the behaviour of pedestrians and cars in a shared environment. This model is divided into three linked layers: global/trajectory planning level, operational/force-based level and diplomatic/rule-based level.

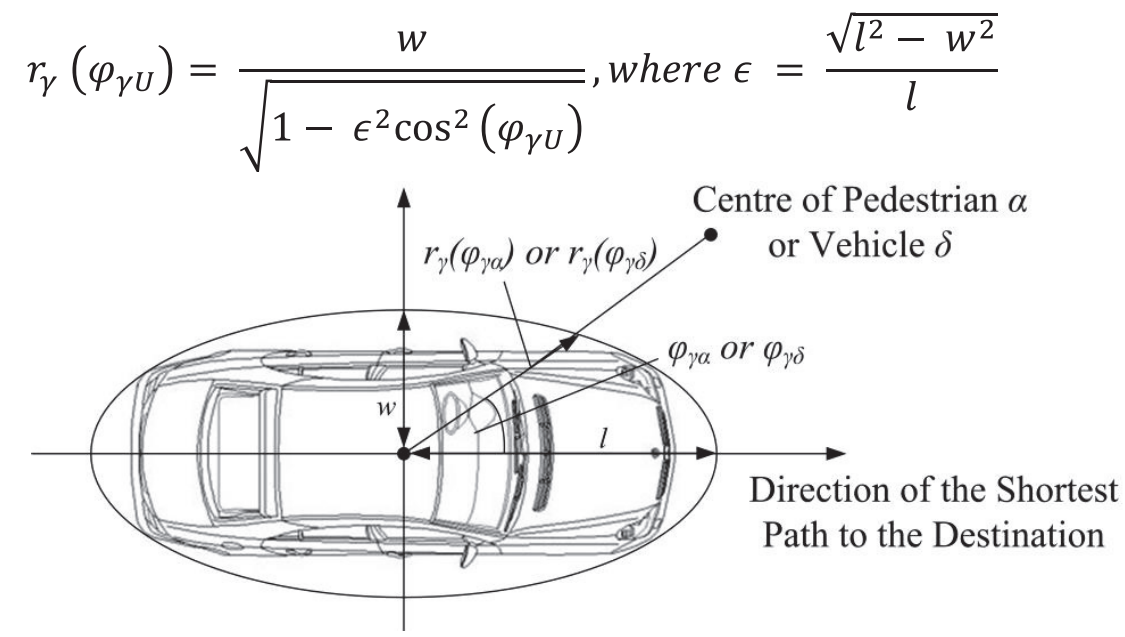

Fig. 2. Vehicle modelling using a geometrical approximation of an ellipse

\section{Trajectory Planning by Distance Map}

As explained in Section 4, the conceptual framework for the proposed mathematical model consists of three interrelated layers. The core model is based on the Social Force Model (SFM) and the other layers are included to resolve issues that cannot be resolved following the SFM exclusively. The first layer, the global trajectory planning layer, introduces a distance map based on the flood fill algorithm to define intermediate destinations between an agent's origin and their destination in order to find the shortest path. This layer is implemented to ensure adequate realism for the force-directed model in the second layer. Agent movements are modelled for shared space areas based on the SFM framework proposed by Helbing and Molnar (1995) and Helbing et al. (2000). The need for the trajectory planning level requires a partial recall of the SFM. In Section 0, the original SFM is extended to different transport modes. The original SFM is extended in Section 6.7 for pedestrians in shared space environment by considering cars in the environment.

\subsection{Fundamentals of the SFM by Helbing, Molnar, Farkas and Vicsek}

In the original SFM for crowds, the motion of pedestrians is influenced by a driving force and by repulsive forces exerted by other agents $\beta$ and obstacles $\mathrm{b}$ when they are close-by. The 
driving force $\boldsymbol{f}_{\alpha}^{0}$ motivates an agent $\alpha$ to walk towards its destination while avoiding other agents and obstacles due to the influence of repulsive forces $\boldsymbol{f}_{\alpha \beta}$ and $\boldsymbol{f}_{\alpha b}$ at close distances. A random fluctuation force $\xi$ is added to the sum of the exerted forces to represent diverse behaviours that cannot be measured. $\xi$ has a Gaussian form and is perpendicular to the desired direction of movement. The sum of all the forces causes the movement towards the direction as defined in Eq. (2) by assuming a mass of $\mathrm{m}=1 \mathrm{~kg}$, the unit mass.

$$
\frac{d \boldsymbol{v}_{\alpha}(t)}{d t}=\boldsymbol{f}_{\alpha}^{0}+\sum_{\beta(\beta \neq \alpha)} \boldsymbol{f}_{\alpha \beta}+\sum_{b} \boldsymbol{f}_{\alpha b}+\boldsymbol{\xi}
$$

The first term of Eq. (2), $\boldsymbol{f}_{\alpha}^{0}$, encourages the agent $\alpha$ to move in a desired direction $\boldsymbol{e}_{\alpha}^{0}$ with the desired speed $v_{\alpha}^{0}$ that is adapted to the actual velocity $\boldsymbol{v}_{\alpha}$ within a certain relaxation time $\tau_{\alpha}$ (Eq. (3)). $\boldsymbol{r}_{\alpha}^{n}$ is the final destination or goal of an agent and $\boldsymbol{r}_{\alpha}$ is the momentary location of the agent.

$$
f_{\alpha}^{0}=\frac{v_{\alpha}^{0} e_{\alpha}^{0}-v_{\alpha}}{\tau_{\alpha}}, \text { where } e_{\alpha}^{0}(\mathrm{t})=\frac{r_{\alpha}^{n}-r_{\alpha}}{\left|r_{\alpha}^{n}-r_{\alpha}\right|}
$$

It is not sufficient, however, to guide a pedestrian or a car only by the final destination. When an agent tries to avoid a fixed obstacle that is located between the position of the agent and the goal, the repulsive force $\boldsymbol{f}_{\alpha b}$ in the SFM will keep a certain offset from the obstacle in close proximity (similar to a wall following algorithm) as shown in Fig. 4 - Trajectory (a). However, road users plan their travel route according to static obstacles, not only within their close proximity, but also within their sight distance. Thus, the calculation of the shortest path to the destination using a distance map and predefining intermediate destinations overcomes this navigation problem.

\subsection{Fundamentals of the Flood Fill Algorithm}

In the flood-fill algorithm, space is divided into separate cells where each cell is assigned a distance value. Flood-fill is an algorithm that determines the area connected to a given cell in a multi-dimensional array. To find the shortest path, the flood-fill algorithm "floods" the area by assigning each cell with a distance value calculated to the nearest cell until reaching the start cell from the destination cell. This will result in a distance map. The flood-fill algorithm requires three parameters: the start cell, target cell, and replacement distance value. Empty cells are set to -1 , the destination cell is set to 0 and a large number (max value) to all obstacle and boundary cells. The algorithm considers all empty cells which are connected to the start cell in the activity area and changes their assigned value to the replacement distance value. The distance values of direct neighbourhood cells are added at each iteration. The process is repeated until the distances are calculated for all cells. The distance value can be calculated by standard metrics (the Manhattan and Chessboard metric) and their combination, called Variant 2. The Manhattan distance between two cells is the sum of the horizontal and vertical segments. Fig. 3 illustrates the transmission of local distance with the Manhattan metric $D^{M}$ with assigned distance values, starting from the cell in the centre. The Chessboard distance $D^{C}$ between two cells is the greatest of the horizontal and vertical segments. The flood-fill algorithm is used to generate an obstacle map, a distance map based on both the Manhattan metric and on the Chessboard metric, and a distance map based on a combination of the Manhattan and Chessboard map as in Eq. (4) (Variant 2 distance map). 


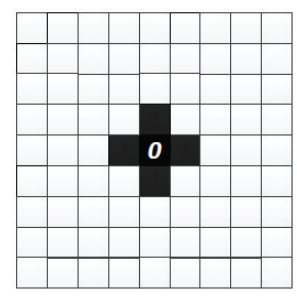

(a) First Iteration

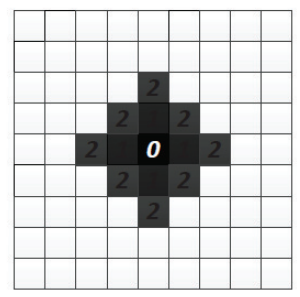

(b) Second Iteration

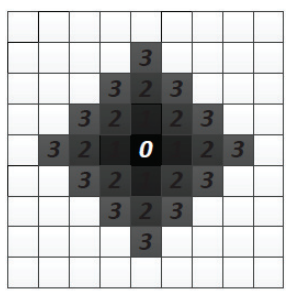

(c) Third Iteration

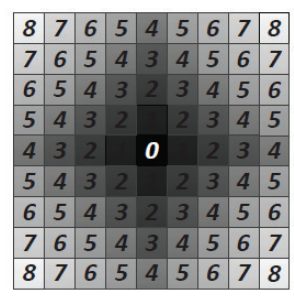

(d) Final Iteration

Fig. 3. Transmission of local distance by Manhattan distance map

\subsection{Calculation of Intermediate Destinations using Flood Fill Algorithm}

In order to define intermediate destinations for each agent in the SFM so as to avoid obstacles in the environment, a global shortest path strategy is presented based on a priori knowledge. In this process, the floor area is divided into cells of $15 \times 15 \mathrm{~cm}^{2}$ in size, the distance values of all the obstacle cells are assigned a large number, with the empty cells being set to zero. Then, a distance map is generated through iterations. The distance values of eight direct neighbourhood cells are added starting from the destination point and finishing at the starting point. This is achieved by calculating the Variant 2 flood fill $\left(D^{V 2}\right)$ based on a combination of Manhattan metric $D^{M}$ and Chessboard metric $D^{C}$ (Kretz, et al., 2008) as in Eq. (4).

$$
D^{V 2}=(\sqrt{2}-1) D^{m}+D^{C}, \text { where }\left\{\begin{array}{c}
D^{M}=\sum_{i}\left|\delta x_{i}\right|+\sum_{i}\left|\delta y_{i}\right| \\
D^{C}=\sum_{i} \max \left(\left|\delta x_{i}\right|,\left|\delta y_{i}\right|\right) \\
D^{m}=D^{M}-D^{C}
\end{array}\right.
$$

This effect is illustrated in Fig. 4 - Trajectory (b). The agent is a single pedestrian. Two static obstacles are positioned intersecting the straight line between the starting point and goal. Without the distance map, the desired destination component of the driving force is dominant until the first boundary enters the interaction range of the agent. After navigating around the first obstacle due to the repulsive forces exerted from the obstacles to the agent, the driving force again dominates until the second obstacle comes within close proximity and then finally reaching the destination. 


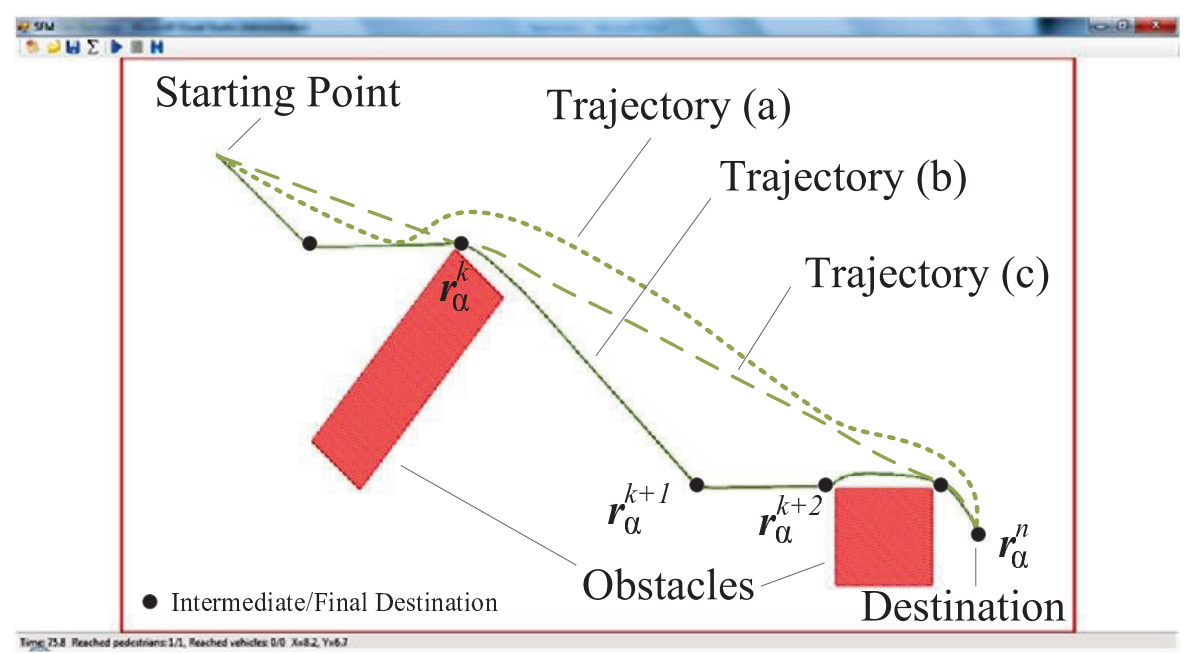

Fig. 4. Obstacle avoidance and way-finding manoeuvres: Trajectory (a) is simulated without a distance map, Trajectory (b) is simulated with a route map and Trajectory (c) is the optimal path simulation

The model's prior geometrical information about the environment and the shortest path to the destination is given before running the simulation. Intermediate destinations are automatically generated for the agents, according to distance map calculations. Some of the calculated intermediate destinations are unnecessary. Let $\boldsymbol{r}_{\alpha}^{k}$ be any intermediate destination while $\boldsymbol{r}_{\alpha}^{n}$ is the final destination (see Fig. 4). In order to reduce the unnecessary intermediate destinations, the line connection between intermediate destinations $\boldsymbol{r}_{\alpha}^{k}$ and $\boldsymbol{r}_{\alpha}^{k+2}$, where $(k+$ $2) \leq n$, is checked for intersections with obstacles. The agent navigates via these intermediate destinations. This is referred to as an obstacle check. If the line does not intersect with any obstacle, the intermediate destination $\boldsymbol{r}_{\alpha}^{k+1}$ will be removed.

The geometrical information about the environment and the shortest path to the destination is given before running the simulation. Intermediate destinations are automatically generated for all agents according to the distance map calculations and collision checks with obstacles as illustrated in Fig. 4 - Trajectory (c). The agent navigates via the intermediate destinations assigned to the vertices of the generated shortest path.

\section{Modelling Motorised Shared Space Users with Respect to Pedestrians using the Social Force Model}

When modelling mixed traffic areas social behaviour and the interactions between cars and pedestrians are the most critical factors to take into consideration. Modelling a situation where pedestrians attempt to cross a road without a crosswalk and other similar behaviours is one of the main challenges when developing a shared space simulation. All agents in the simulation are modelled individually using the microscopic model of social forces in a shared transport system. Hence, the force-based layer includes the original SFM as the framework to simulate and describe shared space users' dynamics. The SFM is extended to different transport modes and diverse forces are introduced to reflect behavioural changes taking place during pedestrians' and drivers' interaction. 


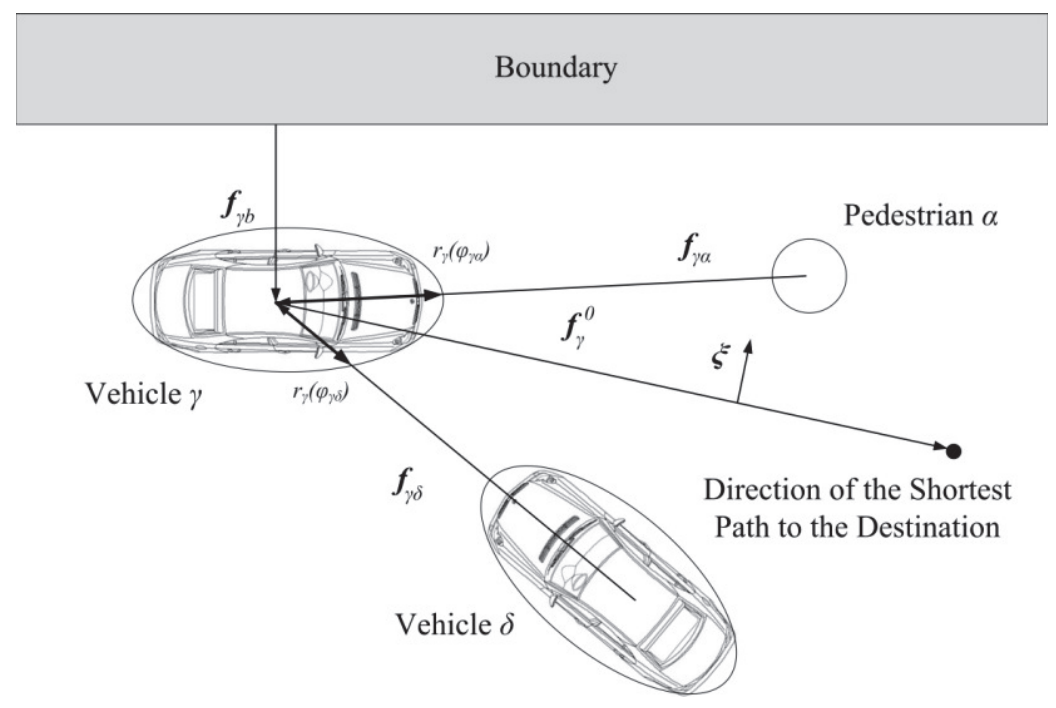

Fig. 5. Force terms exerted on a car from a pedestrian/car/boundary

\subsection{A Microscopic Model for Shared Space Users}

The microscopic model presented in this section is based on the SFM developed by Helbing et al. (2000). The SFM is based on Lewin's (1951) precept that behavioural changes are driven by social forces. Helbing explained this social science idea mathematically and applied it to pedestrian dynamics. Since, in shared space environments, cars and pedestrians move with identical priority, the SFM for pedestrians is here considered and applied also to a model for cars. The new arrangement of a shared space environment integrating vehicles is shown in Fig. 5.

The sum of the force terms exerted to a car $\gamma$ from a pedestrian $\alpha$, a boundary $b$ and another vehicle $\delta$ can be seen in Eq. (5). Each summand is explained in the following sections.

$$
\frac{d v_{\gamma}(t)}{d t}=f_{\gamma}^{0}+\sum_{\alpha} f_{\gamma \alpha}+\sum_{\delta(\delta \neq \gamma)} \boldsymbol{f}_{\gamma \delta}+\sum_{b} \boldsymbol{f}_{\gamma b}+\xi
$$

\subsection{Driving Force}

The driving force of a car is similar to the one applied for pedestrians in the original SFM. This force term describes the motivation of an individual to move towards a certain destination. The driver $\gamma$ is assumed to move in a desired direction of travel with a desired speed $v_{\gamma}^{0}$ that is adapted to the actual velocity $\boldsymbol{v}_{\gamma}$ within a certain relaxation time $\tau_{\gamma}$.

$$
\boldsymbol{f}_{\gamma}^{0}=\frac{v_{\gamma}^{0} \boldsymbol{e}_{\gamma}(t)-\boldsymbol{v}_{\gamma}(t)}{\tau_{\gamma}} \text { where } \boldsymbol{e}_{\gamma}(t)=\frac{\boldsymbol{r}_{\gamma}^{k}-\boldsymbol{r}_{\gamma}}{\left|\boldsymbol{r}_{\gamma}^{k}-\boldsymbol{r}_{\gamma}\right|}
$$

The shortest path to the final destination for car $\gamma$ is defined as a sequence of intermediate destinations. Therefore, the desired direction $\boldsymbol{e}_{\gamma}$ points to the direction of the next intermediate destination $\boldsymbol{r}_{\gamma}^{k}$ on the shortest path to the final destination. This is achieved by calculating a distance map for each agent as explained in Section 4.

\subsection{Interaction Forces Considering the Geometric Model of Cars}

Shared space layouts aim to achieve a constant traffic flow by reducing stop-and-go behaviour (Shearer, 2010), with drivers encouraged to adapt to the behaviour of other shared 
space users. Any deviation from their path to their destination is mainly due to conflict avoiding interactions, therefore. The interaction between a car $\gamma$ either with another car $(U=\delta)$ or with a pedestrian $(U=\alpha)$ is captured by Eq. (7). The socio-psychological force $\boldsymbol{f}_{\gamma U}^{\text {soc }}$ is to keep a certain distance from nearby users. The deceleration force $\boldsymbol{f}_{\gamma \delta}^{\text {following }}$ is to cover the follow-theleader behaviour of drivers if a car is faster than a leading car within a close distance. Assuming no physical contact, which would be equivalent to an accident, vehicles keep a certain distance to other agents. Hence, no physical force is included.

$$
\boldsymbol{f}_{\gamma \delta}(t)=\boldsymbol{f}_{\gamma \delta}^{\mathrm{soc}}(t)+\boldsymbol{f}_{\gamma \delta}^{\text {following }}(t)
$$

To describe the socio-psychological force $\boldsymbol{f}_{\gamma U}^{\text {soc }}$, an exponential function is applied to reflect the role of distance. The repulsive force increases when agents get closer and almost vanishes when they move far away from each other.

$$
\boldsymbol{f}_{\gamma U}^{\mathrm{soc}}(t)=A_{\gamma U} e^{\frac{r_{\gamma U}-d_{\gamma U}}{B_{\gamma U}}} \boldsymbol{n}_{\gamma U} F_{\gamma U}
$$

$\boldsymbol{n}_{\gamma \mathrm{U}}$ is the normalised vector pointing from another road user (car or pedestrian) to the car. $A_{\gamma \mathrm{U}}$ is the constant interaction strength that indicates the amplitude of repulsive forces on agents in the centre mass. The interaction strength affects how quickly the exerted force decreases with distance. $B_{\gamma \mathrm{U}}$ is a constant interaction range which determines the influence of distance on the repulsive force $\boldsymbol{f}_{\gamma \mathrm{U}}^{\mathrm{soc}} \cdot \mathrm{d}_{\gamma \mathrm{U}}$ is the distance between the centre of agents, and $\mathrm{r}_{\gamma \mathrm{U}}$ is the sum of their radii.

Similar to Helbing et al. (2002), the anisotropic character of interactions is included to provide a more realistic form of the forces. Considering that car movement is restricted to change of direction, and that lateral movement is not possible, an effective field of view is included in this form factor $F_{\gamma U}$ (see Eq. (9)). In addition, there is a difference between a pedestrian and a car following another car since the leading car driver not only reacts to the cars in front but also to those behind it. The form factor $F_{\gamma U}$ in Eq. (9) is a dimpled limacon. By varying $\lambda$ within $0 \ll \lambda \ll 1$, the strength of the influence of forces exerted from other agents to a car varies depending on $\varphi_{\gamma U}$ (see Fig. 2) and q.

$$
F_{\gamma U}=\left(\lambda_{\gamma}+\left(1-\lambda_{\gamma}\right) \frac{1+\cos \left(\varphi_{\gamma U}\right)}{2}\right) \cdot q
$$

$\mathrm{q}$ is the 'effective factor' that distinguishes between a car-pedestrian or a car-car interaction. The driver's vision is compared to the effective field of view in Fig. 6. Regarding a car-pedestrian interaction, $\mathrm{q}$ is as in Eq. (10) and as a result, drivers only react to the pedestrians in front of them, as shown in Fig. 7.

$$
\begin{aligned}
& q=1, \text { if }-30^{\circ} \leq \varphi_{\gamma \alpha} \leq 30^{\circ} \\
& q=0, \text { otherwise }
\end{aligned}
$$

Considering a car-car interaction, Eq. (11) can be summarised for q. By varying $\lambda_{\gamma}$, the influence of forces exerted by the cars behind the leading car changes, as illustrated in Fig. 8 .

$$
\begin{gathered}
q=1 \text {, if }\left\{\begin{array}{c}
-30^{\circ} \leq \varphi_{\gamma \delta} \leq 30^{\circ} \text { and } \\
\left(180^{\circ}-30^{\circ}\right) \leq \varphi_{\gamma \delta} \leq\left(180^{\circ}+30^{\circ}\right)
\end{array}\right. \\
q=0, \text { otherwise }
\end{gathered}
$$




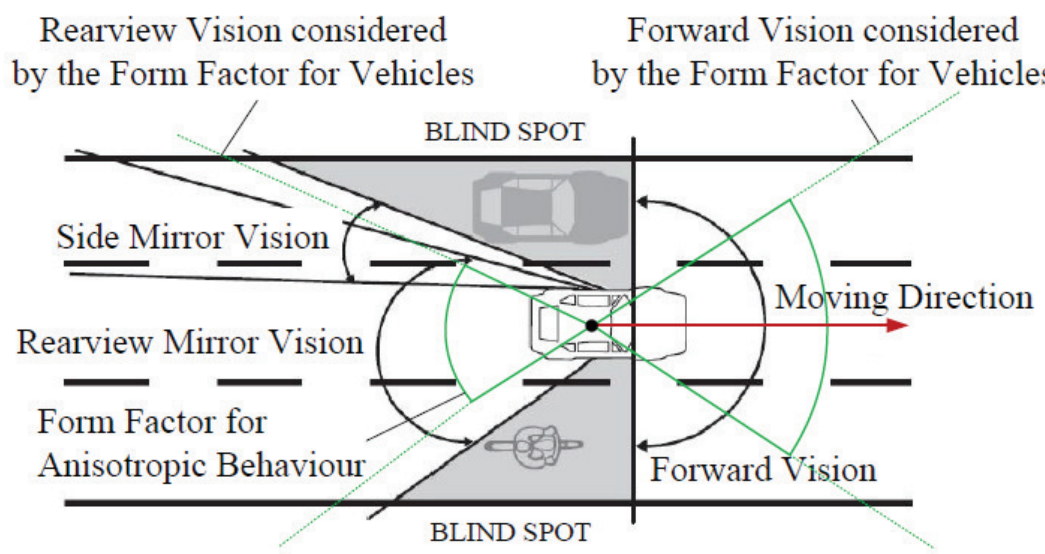

Fig. 6. Effective field of view compared to driver's vision

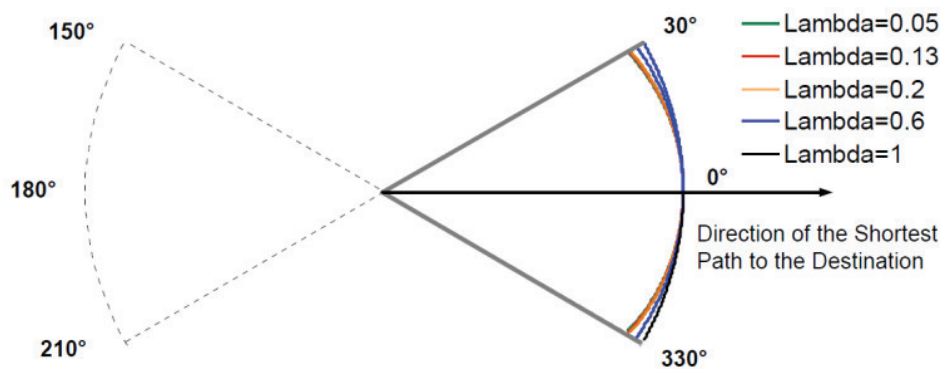

Fig. 7. Effective field of view compared to driver's vision for a car-pedestrian interaction (Eq. (9) and (10))

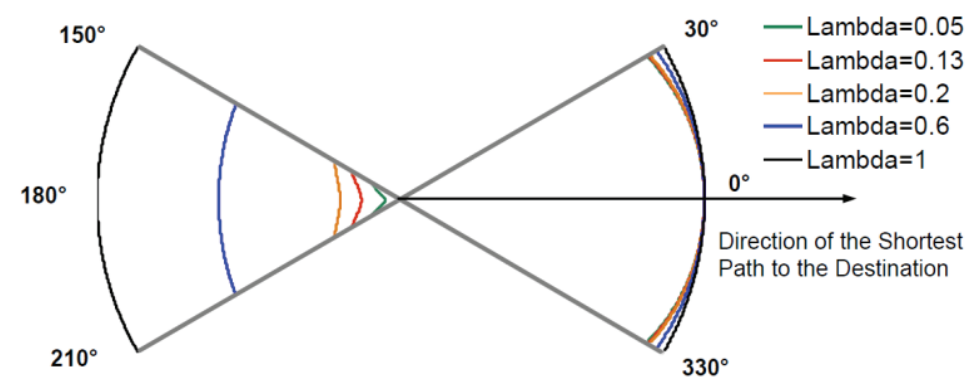

Fig. 8. Effective field of view compared to driver's vision for a car-car interaction (Eq. (9) and (11))

\subsection{Car Following Feature}

As explained in Section 1, shared space schemes involve a single surface with no defined lanes. The philosophy of shared space gives all agents, pedestrians and vehicles the freedom to move in a two dimensional space. However, empirical data gathered from observations of shared space schemes in practice shows that vehicles merge into assumed lanes created by car drivers. This becomes noticeable when a number of cars move towards the same destination within a close distance from each other.

The social force results in overtaking rather than following the leader if an obstructed car is approached by another car from behind. If driver behaviours are modelled using purely social 
forces in cases where the leading car decelerates, the following cars with a higher speed will try to overtake (avoid) the leading car immediately instead of queuing in the assumed lane

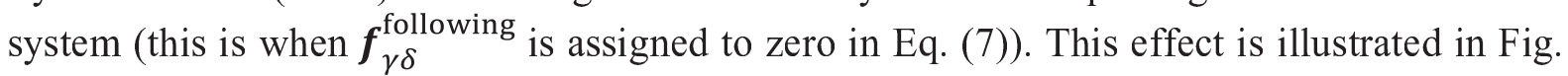
9(a): Two cars travel from starting points on the left to their destination points on the right of the environment. On the way, the leading car is obstructed by two randomly defined pedestrians. Pedestrian 1 crosses the trajectory of the leading car in order to reach his destination point. The desired direction of movement (left to right) of Pedestrian 2 intersects the desired direction of the leading car. The leading car decelerates and changes its desired direction to avoid conflict.

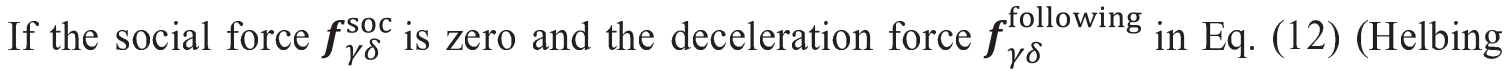
and Tilch, 1998) is applied, a flexible car-following feature is captured. In other words, the following car adapts to the velocity of the car in front as shown in Fig. 9(b). The magnitude of the deceleration force depends on the distance between cars $d_{\gamma \delta}$ considering the velocity dependence safe distance $d\left(v_{\gamma \delta}\right)=d_{\gamma}+T_{\gamma} v_{\gamma}$, velocity differences $\Delta \boldsymbol{v}_{\gamma \delta}$ and braking time $\tau_{\gamma}^{\prime}$. In the speed-dependent safe distance $d\left(v_{\gamma \delta}\right), d_{\gamma}$ is the minimum vehicle distance and $T_{\gamma}$ is the safe time headway. $B_{\gamma \delta}^{\prime}$ and $B_{\gamma \delta}^{\prime \prime}$ are acceleration interaction range and braking interaction range, respectively.

$$
\begin{aligned}
\boldsymbol{f}_{\gamma \delta}^{\text {following }}(t)= & \left(-\frac{v_{\gamma}^{0} \boldsymbol{e}_{\gamma}}{\tau_{\gamma}} e^{\frac{d\left(v_{\gamma \delta}\right)-d_{\gamma \delta}}{B_{\gamma \delta}^{\prime}}}-\frac{\Delta \boldsymbol{v}_{\gamma \delta}}{\tau_{\gamma}^{\prime}} e^{\left.\frac{d\left(v_{\gamma \delta}\right)-d_{\gamma \delta}}{B_{\gamma \delta}^{\prime \prime}} \Theta\left(\Delta v_{\gamma}\right)\right) \cdot p,}\right. \\
& \text { where }\left\{\begin{array}{lc}
\Theta\left(\Delta v_{\gamma}\right)=1, & \text { if }\left(\Delta v_{\gamma}\right)>0 \\
\Theta\left(\Delta v_{\gamma}\right)=0, & \text { otherwise }
\end{array}\right.
\end{aligned}
$$

The car following behaviour should be performed only if the cars are moving confluent. The angle between the desired direction of a driver $\gamma$ and that of the driver in front $\delta$ determines whether their movements are confluent or opposed. The deceleration force is only included $(p=1)$ in the sum of the forces when cars move towards the same destination within a close distance from each other $\left(\left|\varphi_{\gamma \delta}\right|>10^{\circ}\right.$ or $\left.\left|\varphi_{\gamma \delta}+\varphi_{\delta \gamma}-180\right|<10^{\circ}\right)$. The social force is assigned to zero in this case. Otherwise, $p=0$ and the social force will manage the social interaction. 


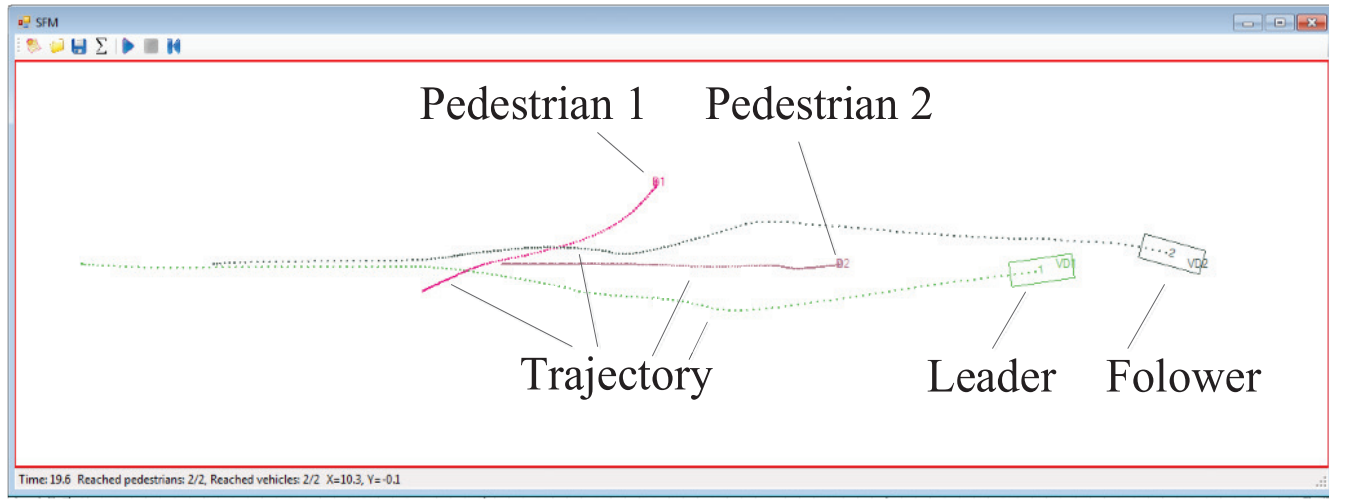

(a)

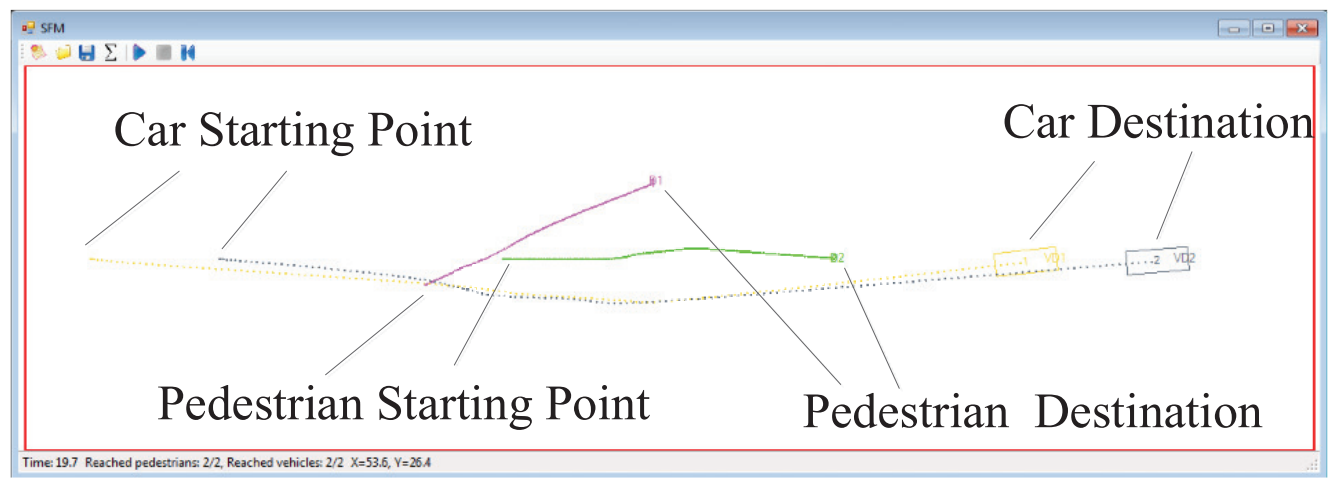

(b)

Fig. 9. Trajectory simulation of an obstructed car and the following car according to (a) social forces and (b) the deceleration force

\subsection{Interaction between Cars and Boundaries/Obstacles}

Considering that cars are not expected to have any physical contact with obstacles, an expression similar to Eq. (8) is defined for car accident avoidance as:

$$
\boldsymbol{f}_{\gamma b}=A_{\gamma b} e^{\frac{r_{\gamma}-d_{\gamma b}}{B_{\gamma b}}} \boldsymbol{n}_{\gamma b} F_{\gamma b}
$$

Here, $\boldsymbol{n}_{\gamma b}$ is the normal vector to the surface of a boundary or obstacle. Also, $d_{\gamma b}$ is the algebraic distance between the centre of car $\gamma$ and the closest vertex of the boundary/obstacle polygon. The distance between a pedestrian and a boundary/obstacle is calculated in the same way. $F_{\gamma b}$ is also the form factor regarding car-boundary interactions similar to car-pedestrian interactions.

\subsection{Fluctuation Term}

The last term in Eq. (14) is the velocity fluctuation due to imperfect driving. The fluctuation term $\xi$ has a Gaussian form based on the observed data in (Schonauer et al., 2012). Also, it is perpendicular to the desired direction of movement $\mathrm{e}_{\gamma}$ and is calculated from the sum of the forces exerted to car $\gamma$ as follows:

$$
\boldsymbol{\xi}=\left\langle\boldsymbol{e}_{\gamma}, \boldsymbol{f}_{\gamma}\right\rangle \mathrm{X} \cdot \boldsymbol{e}_{\gamma}^{\text {norm }}
$$




\subsection{Social Force Model Extension for Pedestrians}

The existence of cars in a shared space environment is expressed by a new socio-repulsive force term $\boldsymbol{f}_{\alpha \gamma}$. This force is exerted by a car to pedestrians. This new force explains the important interaction behaviour of a pedestrian who keeps a certain distance from a nearby car, since no physical interaction should occur. The socio-repulsive force $\boldsymbol{f}_{\alpha \gamma}^{\text {soc }}$ is added to the sum of forces exerted to pedestrians in the shared space model:

$$
\frac{d v_{\alpha}(t)}{d t}=\boldsymbol{f}_{\alpha}^{0}+\sum_{\beta(\beta \neq \alpha)} \boldsymbol{f}_{\alpha \beta}+\sum_{b} \boldsymbol{f}_{\alpha b}+\sum_{\gamma} \boldsymbol{f}_{\alpha \gamma}^{\mathrm{soc}}+\xi
$$

Similar the interaction force among pedestrians in the SFM, an exponential function is applied to pedestrian $\alpha$ to represent the influence of distance between the pedestrian and the neighbourhood car $\gamma$.

$$
\boldsymbol{f}_{\alpha \gamma}^{\mathrm{soc}}=A_{\alpha \gamma} e^{\frac{r_{\alpha \gamma}-d_{\alpha \gamma}}{B_{\alpha \gamma}}} \boldsymbol{n}_{\alpha \gamma} F_{\alpha \gamma}
$$

where $r_{\alpha \gamma}=r_{\alpha}+r_{\gamma} ; d_{\alpha \gamma}$ is the distance between the centre of pedestrian $\alpha$ and car $\gamma ; \boldsymbol{n}_{\alpha \gamma}$ is the normalized vector from car $\gamma$ to pedestrian $\alpha$ and parameters $A_{\alpha \gamma}, B_{\alpha \gamma}$ are to explain the strength and the reaction distance of the exerted force to pedestrian $\alpha$. The form factor $F_{\alpha \gamma}$ is set in Eq. (17) to explain the anisotropic behaviour of pedestrian $\alpha$ when facing car $\gamma$ :

$$
F_{\alpha \gamma}=\lambda_{\alpha}+\left(1-\lambda_{\alpha}\right) \frac{1+\cos \left(\varphi_{\alpha \gamma}\right)}{2}
$$

\section{Rule-based Constraints for Shared Space Users}

In the third layer, the rule-based layer, car motions are limited by the functional relationship between the steering angle and speed (see Section 7). As mentioned earlier, cars do not have any physical force since any physical contact represents an accident. Hence, the third layer explores how potential road conflicts involving cars and pedestrians that might occur when following the SFM exclusively are resolved by the application of an optimised conflict avoidance strategy (a combination of speed change and correction of heading direction) (see Section 0). A frontal conflict avoidance strategy is introduced following the left-hand traffic rule in the UK for car-car interactions.

\subsection{Relation between Steering Angle and Moving Speed}

The maximum steering angle $\psi_{\gamma}$ of cars is mechanically restricted; cars are unable to move laterally. In addition, car drivers must obey a $8.9 \mathrm{~m} / \mathrm{s}$ speed limit and reduce their speed when driving along a curved trajectory. This type of car motion is addressed by defining a relationship between a car's speed and steering angle as shown in Fig. 10, where the steering angle is considered to be in the interval $\left[0, \pm 30^{\circ}\right]$, while $v_{\gamma}(t)$ does not exceed $8.9 \mathrm{~m} / \mathrm{s}$ (shared space speed limit). The lateral or centrifugal acceleration of a car is speed-dependent when driving along a curve. Fig. 10 shows car $\gamma$ and $\psi_{\gamma}$ is the rotation around the centre position of the car. $\mathrm{L}$ is the distance between the front and the rear axle. If the steering angle $\psi_{\gamma}$ is fixed, the car moves around a circle of radius $\rho_{\gamma}$. For very small slip angles and by considering that the normal component of acceleration is dependent on the $\rho_{\gamma}$ and the speed of the car, the 
centrifugal acceleration can be simplified. In addition, Schimdl (2011) reported that the acceptable lateral acceleration is constant below $8.9 \mathrm{~m} / \mathrm{s}$. The assumption is that $\rho_{\text {Rear }}=$ $\rho_{\gamma}$ and $L=2 l$ can be estimated since $\rho_{\gamma}>>2 l$ for our application.

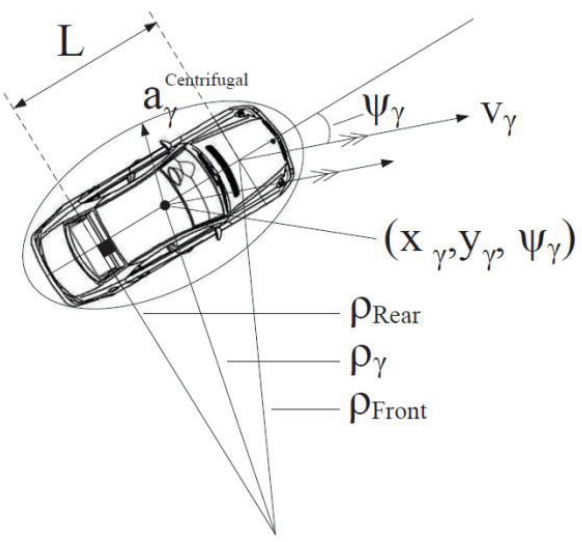

Fig. 10. Parameters of a turning car model in two dimensional space

The relationship between the steering angle and maximum acceptable centrifugal acceleration exerted to a driver in an approximated way is then described in Eq. (18). This function was derived based on vehicle dynamic control by Isermann (2006) and observations from Schimdl (2011).

$$
\psi_{\gamma}=\arctan \frac{2 l \cdot a_{\gamma}^{\text {Centrifugal }}}{\left|v_{\gamma}\right|^{2}}
$$

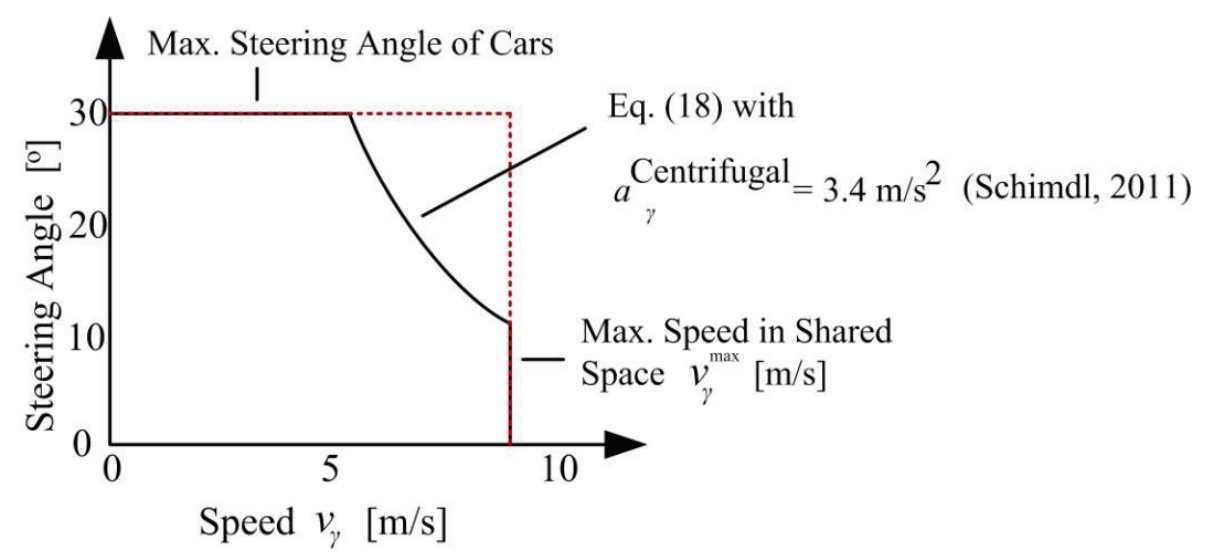

Fig. 11. Car velocity versus steering angle

Fig. 12. shows the trajectory of a single car for the case of a right turning with and without the steering angle constraint. As illustrated in Fig. 12, the turning angle of the car becomes smooth after applying the steering angle constraint for the speed limit of $6 \mathrm{~m} / \mathrm{s}$. 


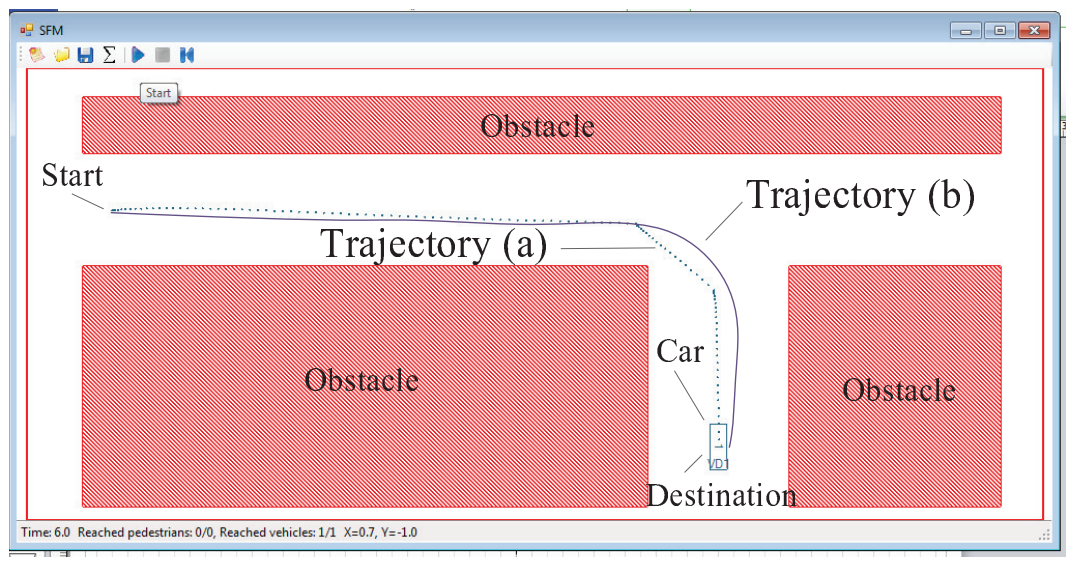

Fig. 12. Driving trajectory simulation of a turning car (a) without steering angle constraint (b) with the steering angle constraint

\subsection{Optimal Manoeuvre for Conflict Avoidance}

A conflict avoidance strategy is implemented for car-pedestrian interactions and - in a modified way - for car-car interactions in order to model left-hand traffic in the UK. To predict whether an interaction between two agents will result to conflict, the agents must be within a certain distance to each other. A conflict is defined by physical contact between two agents at a future time interval. The term 'shadow' is introduced to detect potential conflicts. Conflict avoidance strategies can be classified into speed change, steering change or a combination of both. In this section, agents prevent potential conflicts using a combination of speed and direction change based on their relative position. The aim is then to find the minimum velocity change $\Delta \boldsymbol{v}^{\mathrm{min}}$ for each agent while deviating as little as possible from their desired direction of movement.

In Section 0, a definition of the shadow is presented to predict potential conflicts. An optimisation is applied in order to calculate the minimum velocity change $\Delta \boldsymbol{v}^{\text {min }}$ for carpedestrian interactions. This conflict avoidance strategy is also chosen to model left-hand traffic. The passing preference on the left hand side in the UK for two vehicles driving in opposite directions is described in Section 0.

\subsubsection{Prediction and Resolution of Potential Conflicts}

The conflict avoidance constraints are explained based on the geometrical considerations of two agents. Fig. 13 illustrates the predicted intersecting trajectories of a pedestrian $\left(U_{1}=\alpha\right)$ and a car $\left(U_{2}=\gamma\right)$. The position, direction of movement and initial velocity of pedestrian $\alpha$ and car $\gamma$ are shown in Fig. 13(a). Two lines tangential to pedestrian $\alpha$ and parallel to the velocity difference are indicated in order to assign a section on the desired direction of car $\gamma$. This section is defined as the shadow of pedestrian $\alpha$ along the direction of car $\gamma$ similar to air traffic management systems (Pallottino \& Feron, 2002). A potential conflict is detected as soon as car $\gamma$ intersects the shadow as shown in Fig. 13(b). This is explained mathematically in Eq. (19).

$$
\frac{\left(v_{y, \alpha}-v_{y, \gamma}\right)}{\left(v_{x, \alpha}-v_{x, \gamma}\right)}<\tan \left(\varphi_{\alpha \gamma}\right)
$$

The time $t^{\mathrm{CPA}}$ indicating the period to reach the location of minimum distance $d^{\mathrm{CPA}}$ between the agents at their Closest Point of Approach (CPA) needs to be determined. The minimum distance at the $\mathrm{CPA}$ is $d^{\mathrm{CPA}}=\left|r_{\alpha}\left(t^{\mathrm{CPA}}\right)-r_{\gamma}\left(t^{\mathrm{CPA}}\right)\right|$ and should not be less than 
the sum of their radii $\left(r_{\alpha}+r_{\gamma}\left(\varphi_{\alpha \gamma}\right)\right)$ as shown in Fig. 14. The position of an agent $\alpha$ and $\gamma$ is given by $\left(x_{\alpha}(t), y_{\alpha}(t)\right)$ and $\left(x_{\gamma}(t), y_{\gamma}(t)\right)$. Their time-dependent velocity vectors are $\left(v_{x, \alpha}(t), v_{y, \alpha}(t)\right)$ and $\left(v_{x, \gamma}(t), v_{y, \gamma}(t)\right)$. At any time instance $t$, the distance between the two agents is given by $d(t)=\sqrt{\Delta x(t)^{2}+\Delta y(t)^{2}}$. The time to the minimum distance is now calculated as $t^{\mathrm{CPA}}=-\left(\Delta x \Delta v_{x}+\Delta y \Delta v_{y}\right) /\left(\Delta v_{x}^{2}+\Delta v_{y}^{2}\right)$. The time $t^{\mathrm{CPA}}$ to reach the CPA should be positive (in other words, in the future) and less than a defined higher bound of $t^{\mathrm{CPA} \text {,max }}$ (in other words, in the close future). Moreover, in order to activate the conflict avoidance strategy, the distance to reach the CPA should be less than a certain value (in other words, in the close future).

The conflict avoidance strategy involves the agent with a greater speed starting to accelerate and deviate whereas the other agent decelerates and deviates accordingly. An optimisation is applied to calculate a minimum velocity change $\Delta \boldsymbol{v}^{\min }=\boldsymbol{v}^{\mathrm{opt}}(t)-\boldsymbol{v}(t)$ in order to avoid conflicts. The aim is to optimise the cost function $\mathrm{c}\left(v_{x}^{o p t}(t), v_{y}^{o p t}(t)\right)$ in Eq. (20).

$$
\mathrm{c}\left(v_{x}^{\mathrm{opt}}(t), v_{y}^{\mathrm{opt}}(t)\right)=\left(v_{x}^{\mathrm{opt}}(t)-v_{x}(t)\right)^{2}+\left(v_{y}^{\mathrm{opt}}(t)-v_{y}(t)\right)^{2}
$$

Eq. (20) is to be minimised subject to constraints which are inequalities of variables used in the cost function. Firstly, the optimal speed should be within a defined speed interval $v_{U}^{\min }<$ $v_{U}^{\text {opt }}<v_{U}^{\max }$. Secondly, the minimum distance between the agents at the CPA should be more than the sum of their radii. Thirdly, the distance to reach the CPA should be less than a certain value in order to be considered as a potential conflict.

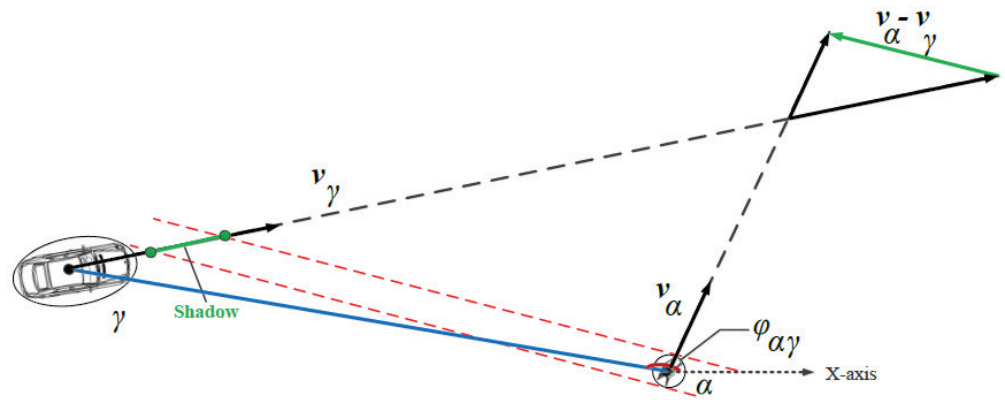

(a)

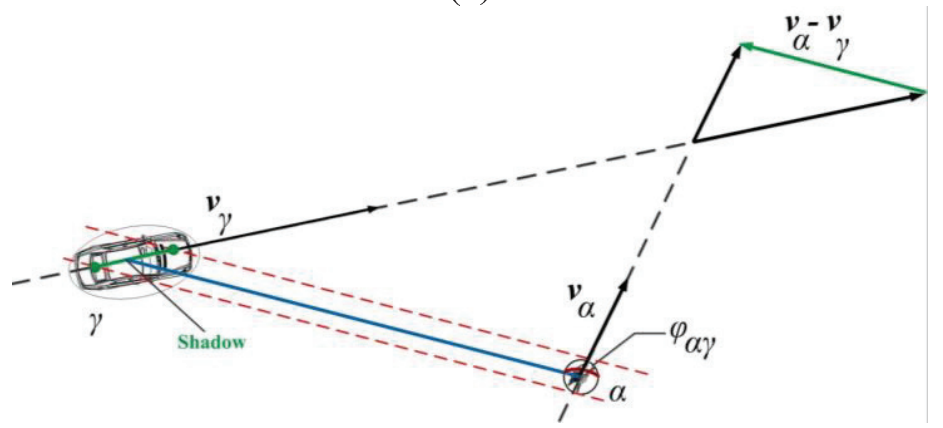

(b)

Fig. 13. Geometric construction for conflict detection: (a) car $\gamma$ does not intersect the shadow generated by pedestrian $\boldsymbol{\alpha}$ (b) car $\boldsymbol{\gamma}$ intersects the shadow generated by pedestrian $\boldsymbol{\alpha}$ 


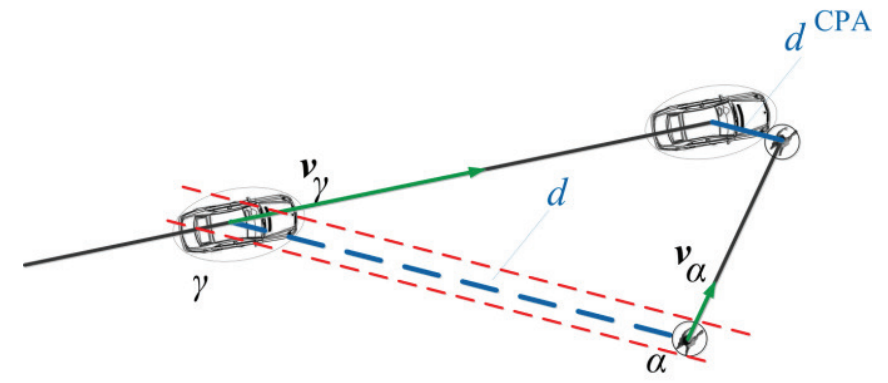

Fig. 14. Closest Point Approach (CPA) illustration between car $\boldsymbol{\gamma}$ and pedestrian $\boldsymbol{\alpha}$

A conflict avoidance force $\boldsymbol{f}_{U}^{\text {conflict }}=\Delta \boldsymbol{v}^{\mathrm{min}} / \tau_{U}$ is calculated and added to the sum of forces. Fig. 15 presents a simulation of the intersecting trajectories of a pedestrian and a car before and after the conflict avoidance force is included. According to social forces, pedestrian $\alpha$ starts decelerating and deviating from the desired direction of movement when within the interaction range $B_{\alpha}$ of the car without prior evaluation of the potential conflict (see Fig. 15(a)). Alternatively, pedestrian $\alpha$ and car $\gamma$ start deviating from their desired direction of movement much earlier as a result of conflict avoidance constraints in Fig. 15(b).

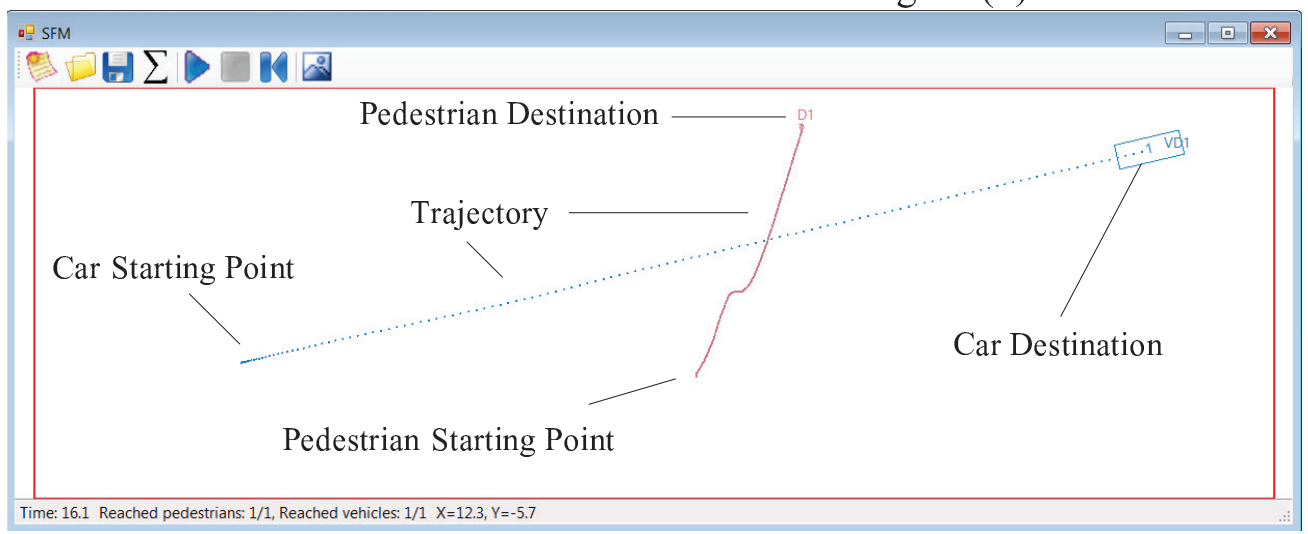

(a)

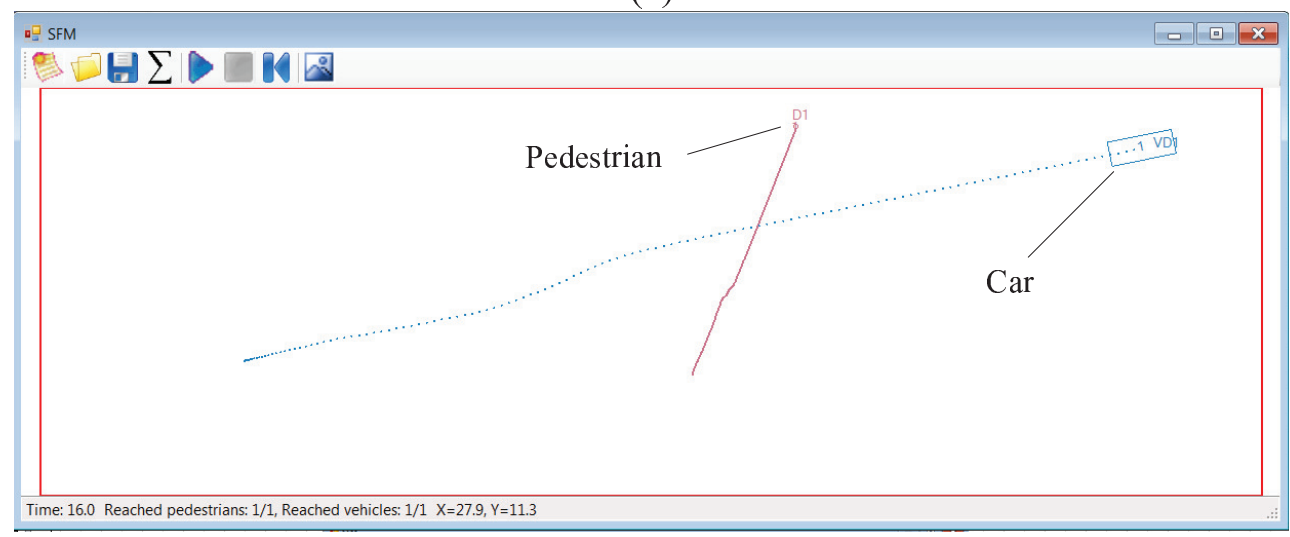

(b)

Fig. 15. Simulation of the interaction between a car and pedestrian (a) without conflict avoidance force and (b) with conflict avoidance force 


\subsubsection{Left Hand Driving Preference in the UK}

The UK is a left-hand traffic country which means that two cars facing each other in opposite directions keep to the left hand side. This general rule avoids confusion between car drivers and decreases the possibility of accidents. A conflict in this situation appears when Eq. (19) and Eq. (21) are met. Eq. (19) detects the conflict and Eq. (21) checks whether they are confluent or opposed.

$$
170^{\circ} \leq \arccos \frac{\boldsymbol{v}_{y}(t) \cdot \boldsymbol{v}_{\delta}(t)}{\left|\boldsymbol{v}_{y}(t)\right| \cdot\left|\boldsymbol{v}_{\delta}(t)\right|} \leq 190^{\circ}
$$

Drivers' preference for the left hand side can be solved by minimising the cost function in Eq. (20) by adding the following constraint to the set of constraints in the conflict avoidance strategy:

$$
0^{o} \leq \arccos \frac{\boldsymbol{v}_{y}(t) \cdot \boldsymbol{v}_{\delta}(t)}{\left|\boldsymbol{v}_{y}(t)\right| \cdot\left|\boldsymbol{v}_{\delta}(t)\right|} \leq 30^{\circ}
$$

The latter condition results in an optimal change of direction by passing the other agent on the left hand side.

\section{Calibration and Simulation}

The individual simulations presented in each of the previous sections show that the proposed mathematical model can be used to describe interactions between shared space users. However, the full representation of reality is strongly dependent on the choice of interaction parameters. During the past years, researchers have progressively calibrated the SFM using empirical data (Helbing, et al., 2000; Johansson, et al., 2008; Steiner, et al., 2007). Since the SFM is extended for shared space environments, the new interaction strengths and ranges need to be calibrated. The interaction strength and interaction range parameters are calibrated and simulated by empirical data using an existing shared space scheme.

\subsection{Case Study}

Bidirectional behaviours of pedestrians and drivers were observed in a shared space street in Brighton, UK (New Road Street, July 2011). The street activity was video recorded with a digital camera (Panasonic HDCHS60 with a resolution of $1920 \times 1080$ pixels) for one hour. The trajectory extractor software developed by Lee (2007) was used to extract the trajectories, velocities and accelerations of road users from video recordings. The video analysis procedure is explained in Lee (2007).

According to the data collected from New Road, pedestrians accelerate and decelerate at the rate of $0.005 \mathrm{~m} / \mathrm{s}^{2}\left(\sigma=0.59 \mathrm{~m} / \mathrm{s}^{2}\right)$ while for cars the rate is about $0.04 \mathrm{~m} / \mathrm{s}^{2}(\sigma=$ $\left.1.22 \mathrm{~m} / \mathrm{s}^{2}\right)$. Shared space users are conscious of each other's behaviour and their small immediate changes of acceleration support this observation. The mean speed that pedestrians achieve during their trip is $1.08 \mathrm{~m} / \mathrm{s}(\sigma=0.7 \mathrm{~m} / \mathrm{s})$ and the mean speed for cars within this shared street is about $2.14 \mathrm{~m} / \mathrm{s}(\sigma=1.79 \mathrm{~m} / \mathrm{s})$ and drivers do not speed up more than $10 \mathrm{~m} / \mathrm{s}$. The paths of pedestrians and cars in New Road are plotted on the camera view in Fig. 16. According to these trajectories, the pedestrians' movement is distributed over the space. Shared space schemes are context dependent their success relying on many factors such as width of the road, design of the environment, and flow of pedestrians and vehicles. The main aim of shared space is essentially to calm the traffic with road users rather than for them. The determination of interaction strength $A$ and interaction range $B$ for pedestrians and drivers 
requires calibration with the obtained real trajectories. The scenarios that exhibited interaction between road users are chosen from New Road for calibration.

\subsection{Calibration Methodology and Results}

In order to determine a reasonable value for the interaction strength $\mathrm{A}$ and the interaction range $\mathrm{B}$, the calibration method of Johansson et al. (2008) is followed: the trajectory of an individual agent is simulated while the agent is reacting to close-by agents (cars or pedestrians) who are moving according to the tracked trajectories. A relative distance error is calculated at the end of each run. The average relative distance errors over all the simulations allow the calculation of the fitness level of that particular parameter set for the interacting agents. The simulation model can then be validated by comparing the trajectories from the calibrated model with the reality for a new given scenario. Fig. 17 presents the resulting fitness values as a function of different combinations of interaction strength $A$ and the interaction range $B$. The local minima show the best fitness for the corresponding choice of $\mathrm{A}$ and $\mathrm{B}$ combinations. Different sets of scenarios are used for pedestrian-pedestrian, pedestrian-car, car-pedestrian and car-car interactions. Combinations of interaction parameters with the highest fitness values are reported in

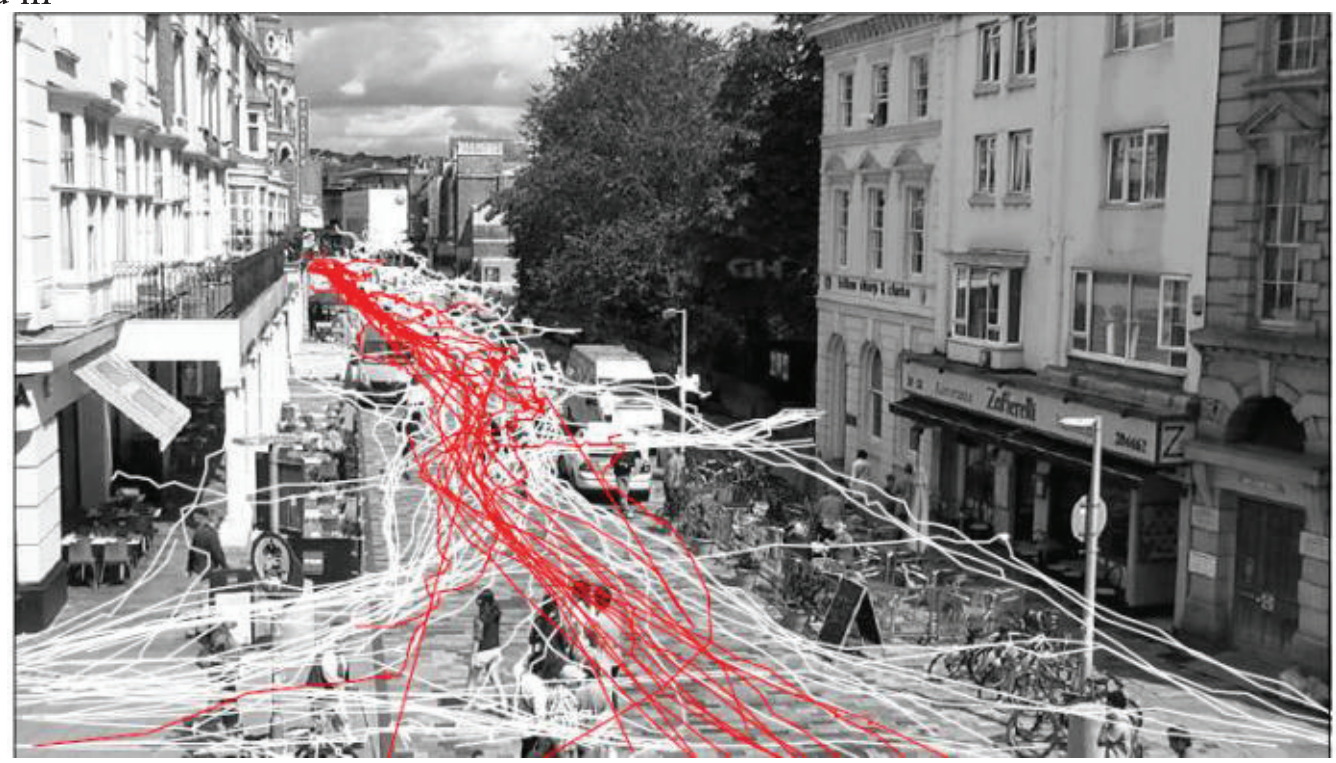

Fig. 16. Trajectories of pedestrians (in white) and cars (in red) in (a) New Road, Brighton

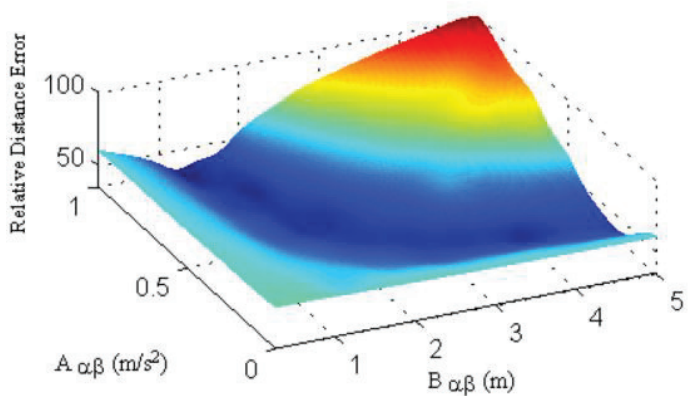

(a)

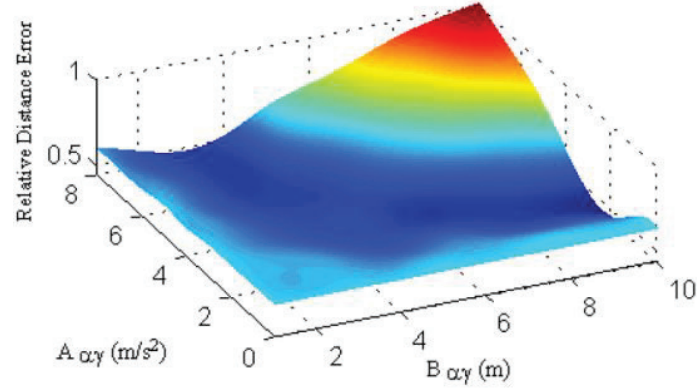

(b) 


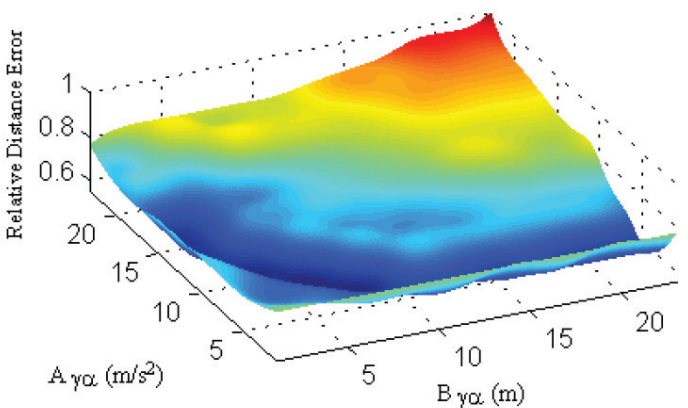

(c)

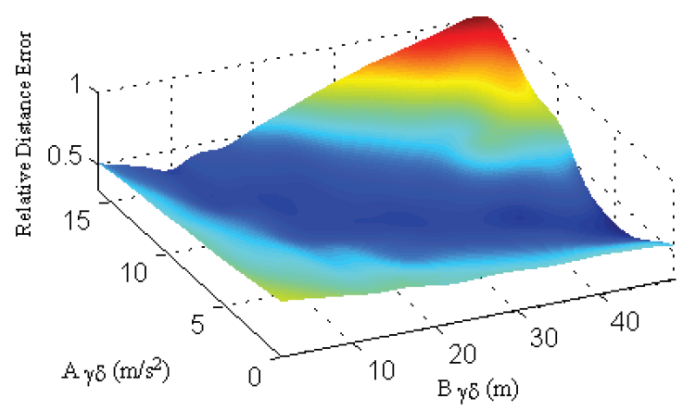

(d)

Fig. 17. The fitness surface based on parameter $A$ and $B$ for (a) pedestrian-pedestrian interactions, (b) pedestrian-car interactions (c) car-pedestrian interactions (d) CarCar Interactions

Table 3 . The travel time and total distance travelled by each agent is compared between the simulated and empirical data. These values are used in the following section for simulating the mathematical model.

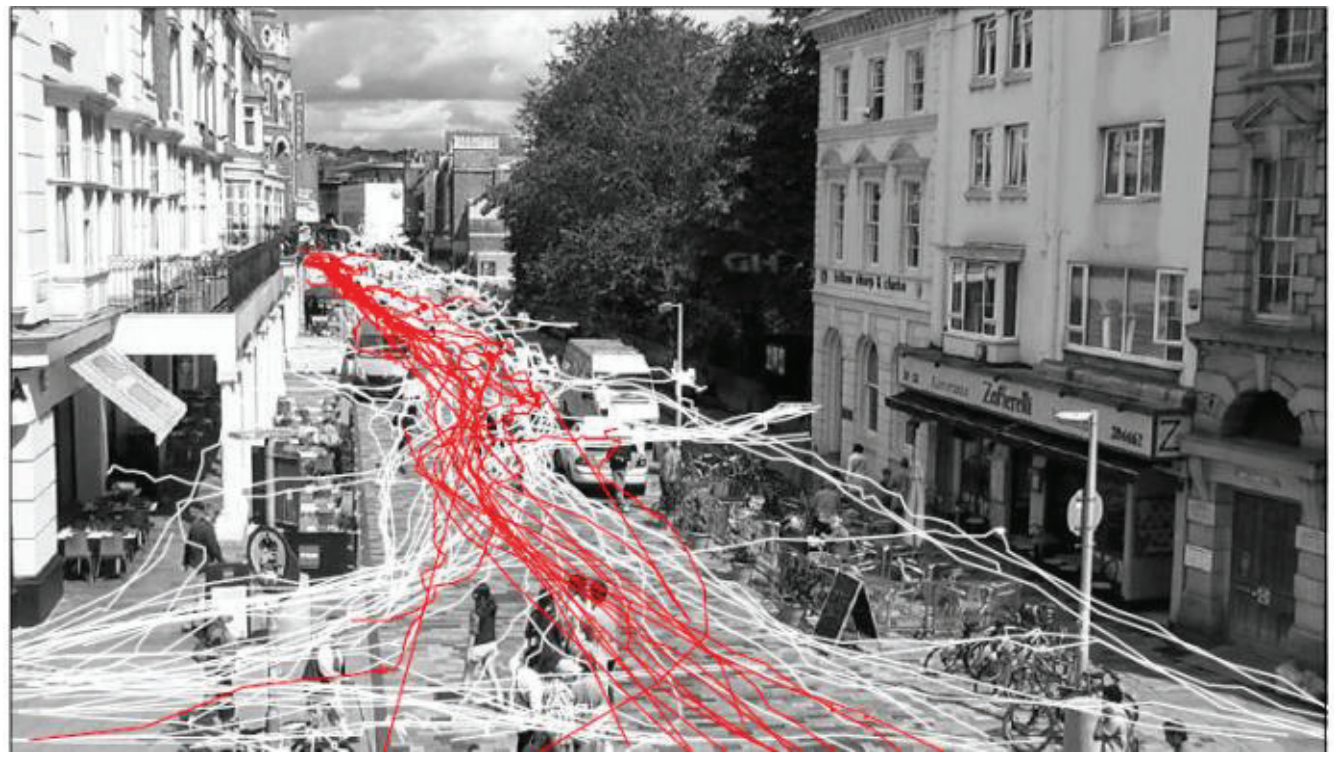

Fig. 16. Trajectories of pedestrians (in white) and cars (in red) in (a) New Road, Brighton

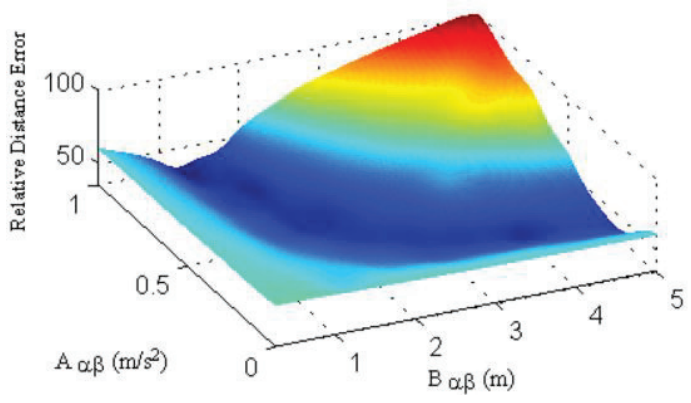

(a)

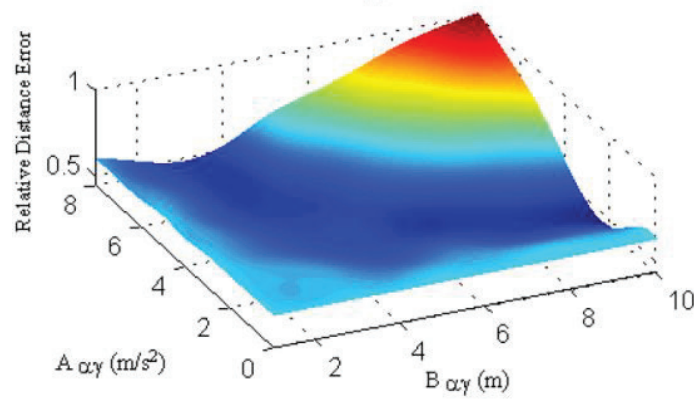

(b) 


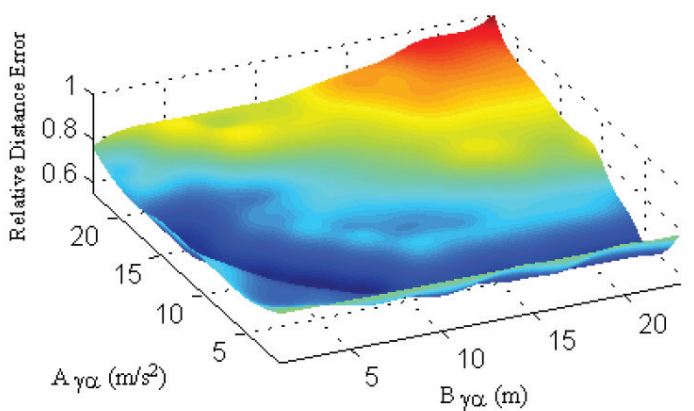

(c)

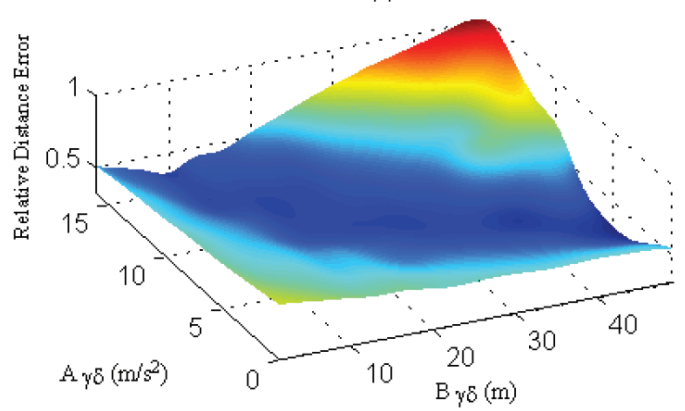

(d)

Fig. 17. The fitness surface based on parameter $A$ and $B$ for (a) pedestrian-pedestrian interactions, (b) pedestrian-car interactions (c) car-pedestrian interactions (d) CarCar Interactions

Table 3

List of parameters for the shared space model

\begin{tabular}{lllr}
\hline Interacting road users & $A\left[\mathrm{~m} / \mathrm{s}^{2}\right]$ & $B[\mathrm{~m}]$ & Fitness \\
\hline Pedestrian-pedestrian & {$[0.7 \pm 0.25]$} & {$[2.25 \pm 1]$} & 0.43 \\
Pedestrian-car & {$[5 \pm 1]$} & {$[3 \pm 1]$} & 0.49 \\
Car-pedestrian & {$[6 \pm 1]$} & {$[5 \pm 1]$} & 0.53 \\
Car-car & {$[8 \pm 1]$} & {$[12 \pm 1]$} & 0.40 \\
\hline
\end{tabular}

\subsection{Simulation Scenarios}

A system analysis of pedestrian and car flow on New Road during peak hours is demonstrated in Fig. 18. An observation of a pedestrian crossing count of up to 1200 per hour and a traffic volume of about 180 per hour is simulated for an area of $13 \times 17 \mathrm{~m}^{2}$ in size. The simulation is defined according to the layout of New Road. Fig. 19 illustrates the trajectories of pedestrians and cars during a time period of $300 \mathrm{~s}$ for real data. The speed distribution of pedestrians and cars according to empirical data and simulation results is shown in Fig. 20.

This simulation includes cars of an average size, $w=1.8 \mathrm{~m}$ and $l=4.8 \mathrm{~m}$. Following Helbing et al. (2000), pedestrians are assumed to have an average shoulder width of $0.5 \mathrm{~m}$ according to Helbing et al. (2000). The desired speeds are set to $v_{\alpha}^{0}=1.3 / \mathrm{s}$ and $v_{\gamma}^{0}=$ $8.33 \mathrm{~m} / \mathrm{s}$ according to the maximum desired speed of pedestrian and the speed limit in shared space schemes. The relaxation time determines how quickly a road user changes its velocity to the desired velocity and they are assigned as $\tau_{\alpha}=0.3 \mathrm{~s}$ and $\tau_{\gamma}=2.4 \mathrm{~s}$. The relaxation time of pedestrians specifies the elapsed time until $63 \%$ of the desired velocity is reached. Maximum acceleration and deceleration limits are assigned to road users based on the observed data on New Road (see Section 23). Cars' braking time and the safety distances are $\tau_{\gamma}^{\prime}=0.77 \mathrm{~s}$ and $d_{\gamma \delta}=1.38 \mathrm{~m}$ as in Helbing and Tilch (1998). The anisotropic character of pedestrian and drivers (the form factor) is set to 0.2 so that interactions outside of the field of view have little effect on the forces. The mathematical model is implemented in Visual C\#. Fig. 18 shows a system analysis of pedestrian and car flow on New Road. Fig. 19 illustrates the trajectories of pedestrians and cars during a time period for real data and simulation. Fig. 20 shows the analysis of the speed distribution for all modes. The speed distribution of the simulated environment and the observed data with their mean and $\sigma$ are presented and they match closely. 


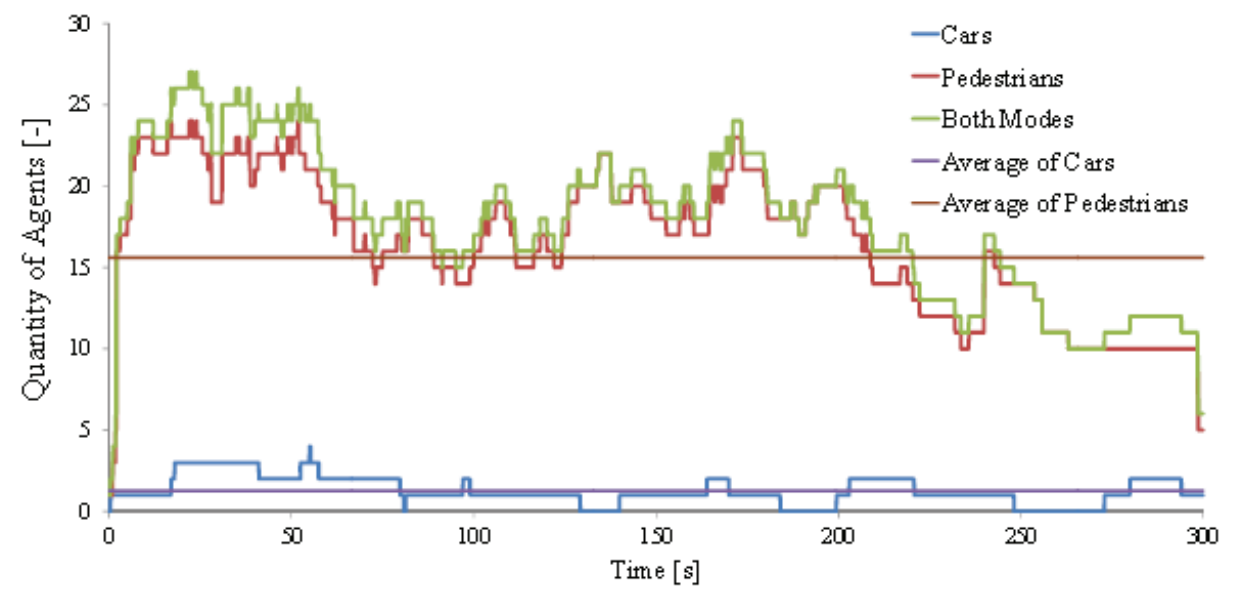

Fig. 18. Traffic demand of tracked road users on New Road (Brighton, UK)

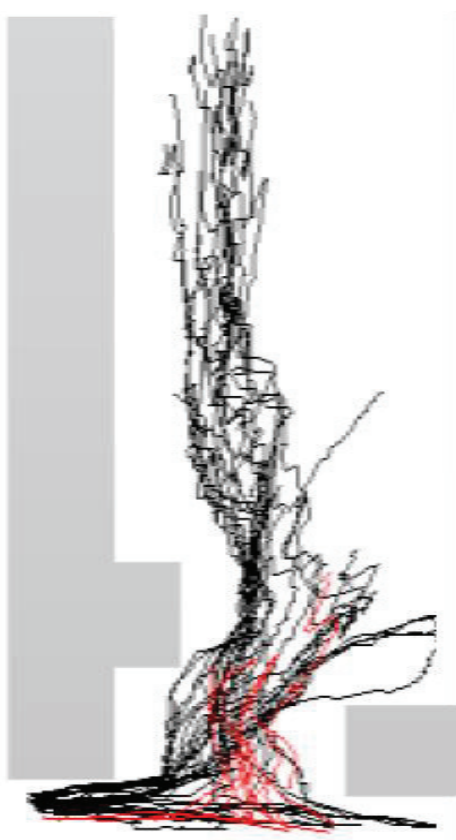

(a)

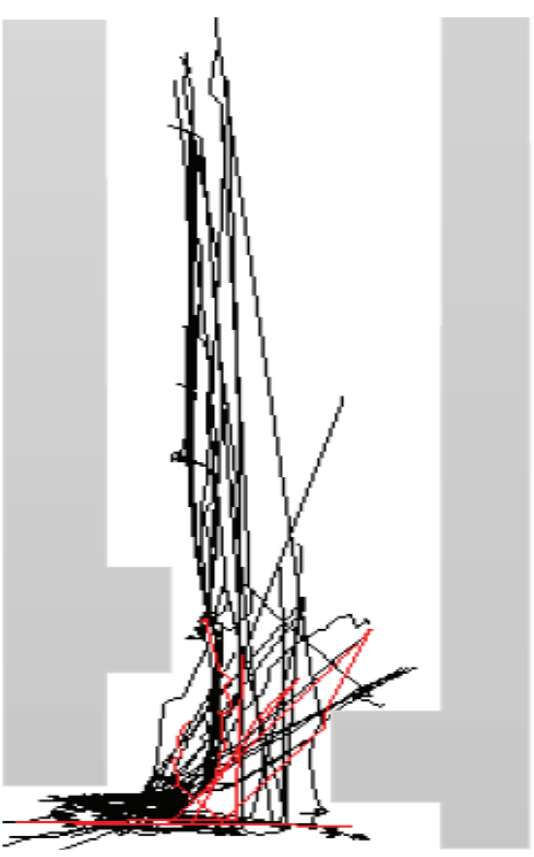

(b)

Fig. 19. Pedestrian (black) and car (red) trajectories on New Road (Brighton, UK) from (a) real data and (b) simulation 


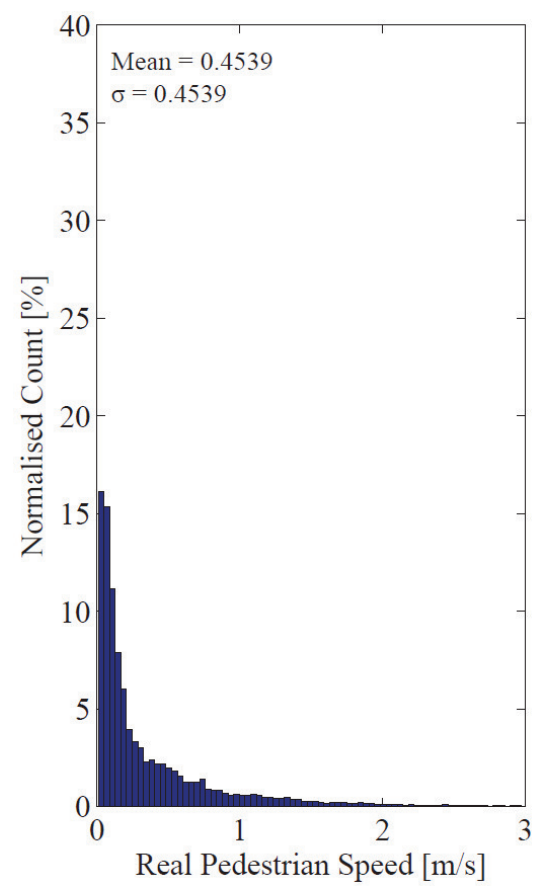

(a)

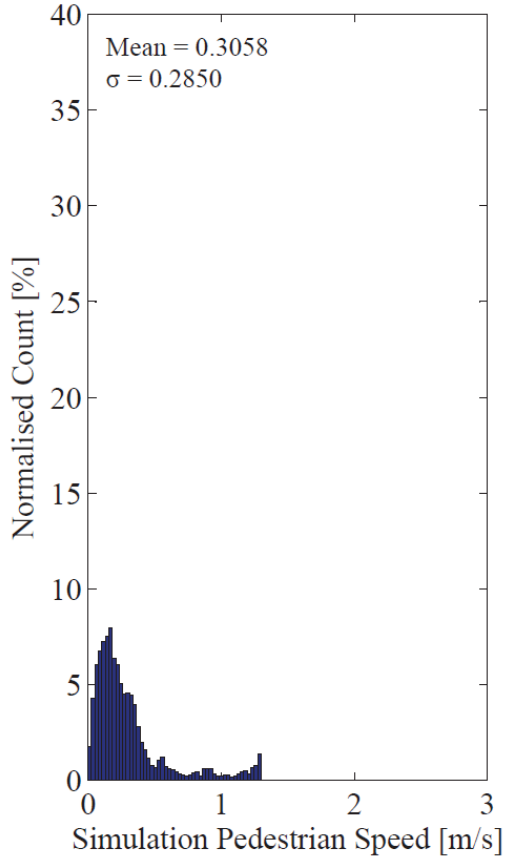

(c)

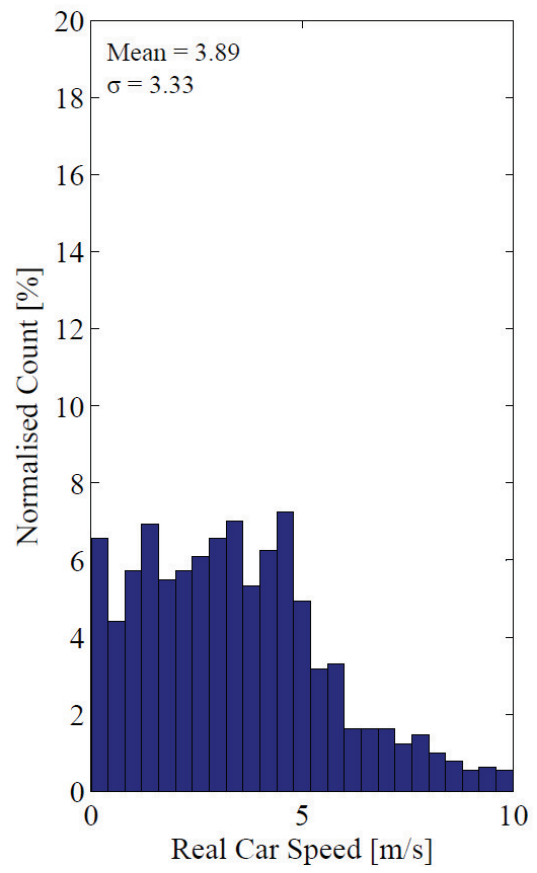

(b)

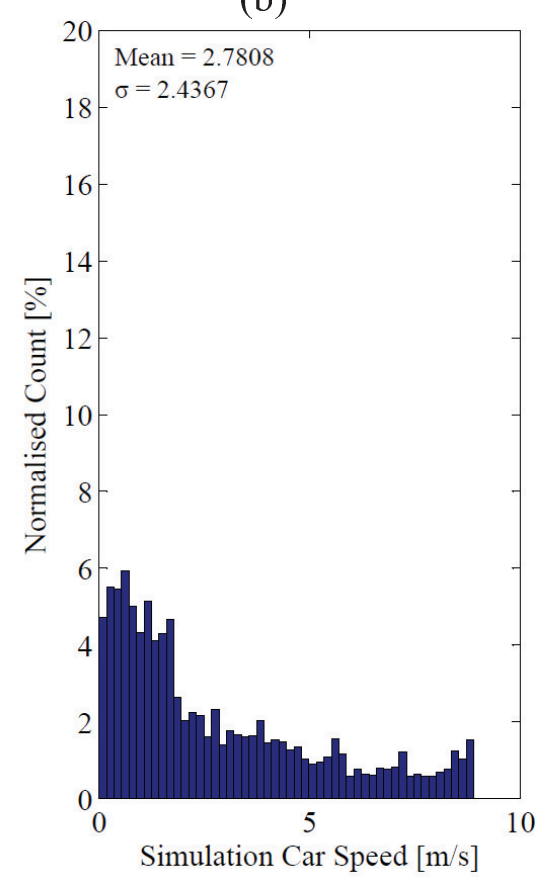

(d)

Fig. 20. Speed histograms of pedestrians and cars on New Road (Brighton, UK) according to (a)-(b) Empirical data and (c)-(d) Simulation results

\section{Further Research}

This simulation platform can be used by researchers to further implement human-like behaviours. As there are many external factors influencing the behaviour of shared space users such as weather, culture, the location of the shared space (city, urban/countryside), shared space configurations, traffic composition, flows (both size and direction), this generic model provides a huge scope for future research. Calibration is an ongoing process and it is always a good idea to test more widely. The calibration process can be fine-tuned to both improve its accuracy and 
its ability to represent different categories of road users and design configurations. It would be interesting, for instance, to adjust desired speeds, relaxation times and interaction parameters in order to reproduce the different behavioural patterns typical in countries and contexts or to analyse different shared space geometries. One can specify typical parameter values for aggressive and conservative drivers or patient and reckless pedestrians as in Zhang and Chang (2011), and to simulate a shared space area with the initial and boundary conditions of interest. These are each interesting challenges for future research. It should be noted that the safety was not the scope of this paper. Thus, safety investigation of the presented shared space model with respect to driving rules and constraints that impact road users' behaviour is left for further studies. It would also be interesting to evaluate the mathematical model for cars presented in this paper for classical traffic characteristics, such as stop-and-go traffic and oscillations when the density of pedestrians and cars increases to a maximum. Extending the new mathematical model to include 2-wheeled motorised and non-motorised vehicles would also strengthen the model, since these modes are known to make use of shared space areas and this additional diversity increases the challenge of simulating shared space schemes. In addition, the influence of the geometrical model of pedestrians and vehicles on the performance of the model needs to be subject to further research. The proposed microsimulation aims to get the design closer to the system optimal. Defining the system optimal and its key performance indicators to measure the extent to which multiple objectives are satisfied in the achieved optimal solution remains to be further studied. Furthermore, the city centres where conventional engineering solutions have been significantly implemented, readjusting the relationship between car and pedestrian movements and removing traffic signals to apply shared space design principles is perhaps inevitable. Since one of the aims of the proposed shared space simulation is to get shared space design closer to the system optimal, further studies is required to describe the system optimal and define its measurement methods.

\section{Conclusions}

A new three-layer mathematical model is presented in this paper to simulate the interactions between road users of a shared space area by giving equal priority to pedestrians and vehicles alike. Observed behaviours were successfully implemented in order to realistically describe pedestrian and vehicle movements. The calibration for interaction parameters was undertaken using the tracked behaviour of pedestrians and cars on New Road (Brighton, UK). This model has been implemented in a simulation platform capable of modelling agent behaviours, conflict detection and resolution, and visualisation.

This simulation tool is recommended to be used by urban designers for visualising the trajectories of pedestrians and cars, extract flow and density relationships in the design stage of new shared space areas so as to achieve solutions for optimal design features before implementation. Road users' speed and traffic participants' distance from each other provide information about potential spots for conflicts and being able to identify these in advance will enable well-designed shared space areas to avoid peaks of density and pressure at critical locations.

Public and local authorities can gain more knowledge about how pedestrians and drivers interact with each other in shared spaces compared to traditional street layouts. Availability of data about proposed shared space schemes will help with the assessment of urban design concepts as well as with education of the public concerning their suitability. Last, but not least, scientists and engineers can make emission and exposure assessments for new street designs. The paper concluded by identifying avenues for further work. 


\section{Acknowledgements}

The authors are thankful to NEARCTIS for partially supporting the research described in this paper under grant number 224272. In addition, many thanks to Dr. Tzu-Chang Lee from the Department of Urban Planning, National Cheng Kung University, Taiwan for supporting this study by allowing the authors to use his Trajectory Extractor software.

\section{References}

Alexandersson, S. \& Johansson, E., 2013. Pedestrians in microscopic traffic simulation. In: Master's thesis. s.1.:Chalmers University of Technology.

Anvari, B., Bell, M., Angeloudis, P. \& Ochieng, W., 2014. Long-range collision avoidance for shared space simulation based on social forces. s.l., nternational Conference on Pedestrian and Evacuation Dynamics.

Anvari, B. et al., 2012. Shared space simulation based on social forces and distance potential field. s.l., International Conference on Pedestrian and Evacuation Dynamics.

Anvari, B., n.d. Mathematical model for driver and pedestrian interaction in shared space environments, 2012: Universities Transport Study (UTSG).

Ben-Joseph, B., 1995. Changing the residential street scene: Adapting the shared street (woonerf) concept to the suburban environment. Journal of the American Planning Association, 61(4), pp. 504-515.

Bonisch, C. \& Kretz, T., 2009. Simulation of pedestrians crossing a street. s.1., In Proceedings of the 8th International Conference on Traffic and Granular Flow.

Chraibi, M., Seyfried, A. \& Schadschneider, A., 2010. Creating shared public space in the contested city: The role of urban design. Physical Review - Part E, 82(4), p. 046111.

Clayden, A., Mckoy, K. \& Wild, A., 2006. Improving residential liveability in the UK: Home Zones and alternative approaches. Journal of Urban Design, 11(1), pp. 55-71.

Department for Communities and Local Government-UK, 2012. Re-imagining urban spaces to help revitalise our high streets, s.1.: The Portas Review.

Department for Transport of UK, 2011. Shared space, s.1.: Local Transport Note 1/11, The Stationery Office.

Dumbaugh, E. \& Li, W., 2011. Designing for the safety of pedestrians, cyclists, and motorists in urban environments. Journal of the American Planning Association, 77(1), pp. 69-88.

Franca, R., Marietto, M., Santana, W. \& Kobayashi, G., 2009. An agent-based simulation model for pedestrian unidirectional movement. s.l., In Applications of Digital Information and Web Technologies, pp. 375-379.

Gaffikin, F., Mceldowney, M. \& Strettett, K., 2010. Generalized centrifugal-force model for pedestrian dynamics. Journal of Urban Design, 15(4), pp. 493-513.

Gaventa, J., 2004. Representation, community leadership and participation: citizen involvement in neighbourhood renewal and local governance, s.l.: Prepared for the Neighbourhood Renewal Unit Office of Deputy Prime Minister, Technical. Report - Institute of Development Studies.

Gerlach, J. \& Methorst, R., 2009. Sense and non-sense about shared space - For an objective view of a popular planning concept. s.l., s.n., pp. 36-45.

Guide Dogs and University College London, 2007. Testing proposed delineators to demarcate pedestrian paths in a shared space environment: Report of design trials conducted at University College London Pedestrian Accessibility and Movement Environment Laboratory (PAMELA), s.l.: s.n.

Hamilton-Baillie, B., 2008. Towards shared space. Urban Design International, 13(2), pp. 130-138. 
Helbing, D., Farkas, I., Molnar, P. \& Viscek, T., 2002. Simulation of pedestrian crowds in normal and evacuations situations. s.1., Pedestrian and Evacuation Dynamics, pp. 21-58.

Helbing, D., Farkas, I. \& Vicsek, T., 2000. Simulating dynamical features of escape panic. Nature, Volume 407, pp. 407-487.

Helbing, D., Jiang, R. \& Treiber, M., 2005. Analytical investigation of oscillations in intersecting flows of pedestrian and vehicle traffic. Physical Review - Part E, 72(4), pp. 0461301-04613010.

Helbing, D. \& Molnar, O., 1995. Social force model for pedestrian dynamics. Physical Review - Part E, Volume 51, pp. 4282-4286.

Helbing, D. \& Tilch, B., 1998. Generalized force model of traffic dynamics. Physical Review - Part E, Volume 58, pp. 133-138.

Isermann, R., 2006. Fahrdynamik-Regelung: Modellbildung, Fahrerassistenzsysteme, s.l.: Mechatronik, Vieweg und Teubner Verlag.

Ishaque, M. \& Noland, R., 2007. Trade-offs between vehicular and pedestrian traffic using microsimulation methods. Transport Policy, Volume 14, pp. 124-138.

Jasti, V. \& Higgs, C., 2005. A lattice-based cellular automata modeling approach for granular flow lubrication. Journal of Tribology, 128(2), pp. 358-364.

Jenks, M., 1983. Residential roads researched. Architects' Journal, Volume 177, pp. 46-49.

Jiang, R. \& Wu, Q., 2006. Interaction between vehicle and pedestrians in a narrow channel. Physica A: Statistical Mechanics and its Applications, 364(1), pp. 239-246.

Jiang, R. \& Wu, Q., 2006. Interaction between vehicle and pedestrians in a narrow channel. Physica A: Statistical Mechanics and its Applications, 364(1), pp. 239-246.

Jian, M., Siuming, L., Xuan, X. \& Weiguo, S., 2011. Dynamic features of pedestrian-vehicle counter flow conflicts. s.1., Third International Conference on Transportation Engineering (ICTE), pp. 697-702.

Johansson, A., Helbing, D. \& Shukla, P., 2008. Specification of the social force pedestrian model by evolutionary adjustment to video tracking data. Advances in Complex Systems, Volume 10, pp. 271-288.

Kaparias, I. et al., 2012. Analysing the perceptions of pedestrians and drivers to shared space.

Transportation Research Part F: Traffic Psychology and Behaviour, 15(3), pp. 297-310.

Kretz, T., Bonisch, C. \& Vortisch, P., 2008. Comparison of various methods for the calculation of the distance potential field. s.l., Pedestrian and Evacuation Dynamics, pp. 335-346.

Lakoba, T., Kaup, D. \& Finkelstein, N., 2005. Modifications of the Helbing-Molnar-Farkas-Vicsek social force model for pedestrian evolution. Simulation, 81(5), pp. 339-352.

Lee, T., 2007. An agent-based model to simulate motorcycle behaviour in mixed traffic flow. s.1.:PhD thesis, Centre of Transport Studies, Imperial College London..

Lewin, K., 1951. Field theory in social science: Selected theoretical papers. s.1.:York: Harper \& Row.

Li, X., Yan, X., Li, X. \& Wang, J., 2012. Using Cellular Automata to investigate pedestrian conflicts with vehicles in crosswalk at signalized intersection. s.l., Discrete Dynamics in Nature and Society 2012, pp. 1-16.

Luca, O., Gaman, F. \& Singureanu, O., 2012. Coping with congestion: Shared space. Theoretical and Empirical Researches in Urban Management, 7(4), pp. 53-62.

Melia, S. \& Hamilton-Baillie, B., 2012. Sharing or separation: Which way for streets of the future? [Interview] 2012.

Monderman, H., 2007. Designing shared space [Interview] 2007.

Ottomanelli, M., Iannucci, G. \& Sassanelli, D., 2012. Simplified pedestrians-vehicles interaction model at road crossings based on discrete events system. Transportation Research Record: Journal of the Transportation Research Board, 2316(1), pp. 58-68. 
Pallottino, L. \& Feron, E., 2002. Conflict resolution problems for air traffic management systems solved with mixed integer programmingConflict resolution problems for air traffic management systems solved with mixed integer programming. Intelligent Transportation Systems, IEEE Transactions, 3(1), pp. 3-11.

Parisi, D. \& Dorso, C., 2007. Morphological and dynamical aspects of the room evacuation process. Physica A: Statistical Mechanics and its Applications, 385(1), pp. 343-355.

Pretto, C., Cybis, H. \& Jacobsen, A., 2011. A multi-layer simulation model for vehicle And pedestrian interaction. s.1., Transportation Research Board 90th Annual Meeting.

Quimby, A. \& Castle, J., 2006. A review of simplified streetscape schemes, s.1.: Transport for London, Street Management Division.

Quimby, A. \& Castle, J., 2006. A review of simplified streetscape schemes, s.l.: Transport for London, Street Management Division.

Schmidl, S., 2011. Analysis of driving performance data in various curve radii on dry roads, s.l.: Master's thesis, University of Agricultural Sciences Vienna.

Schonauer, R. et al., 2012. Modeling concepts for mixed traffic. Transportation Research Record: Journal of the Transportation Research Board, 2316(1), pp. 114-121.

Schonauer, R., Stubenschrott, M., Schrom-Feiertag, H. \& Mensik, K., 2012. Social and spatial behaviour in shared spaces. s.l., In Proceedings of REAL CORP.

Shearer, D., 2010. Shared spaces in New Zealand urban areas, s.1.: Master's thesis, School of Surveying/Te Kura Kairuri.

Southworth, M. \& Ben-Joseph, E., 2003. Streets and the shaping of towns and cities. Washington, D.C: Island Press.

Southworth, M. \& Ben-Joseph, E., 2003. Streets and the shaping of towns and cities. s.1.:Washington, D.C: Island Press.

Steiner, A., Philipp, M. \& Schmid, A., 2007. Parameter estimation for a pedestrian simulation model. s.1., 7th Swiss Transport Research Conference.

Suh, W. et al., 2013. Modelling pedestrian crossing activities in an urban environment using microscopic traffic simulation. Simulation: Transactions of the Society for Modeling and Simulation International, 0(0), pp. 1-12.

Sutcliffe, D., 2009. Shared space and naked intersections - 2008 Municipal engineering foundation victoria overseas study tour, s.l.: Central Goldfields Shire.

Waizman, G., Shoval, S. \& Benenson, I., 2012. Micro-simulation model for assessing the risk of vehicle-pedestrian road accidents. s.1., In Proceedings of the 7th Workshop on Agent in Traffic and Transportation.

Yu, W., Chen, R., Dong, L. \& Dai, S., 2005. Centrifugal force model for pedestrian dynamics. Physical Review - Part E, 72(2), p. 026112.

Zhang, H. \& Kim, T., 2005. A car-following theory for multiphase vehicular traffic flow. Transportation Research Part B, 39(5), pp. 385-399.

Zhang, X. \& Chang, G., 2010. Optimal control strategies for massive vehicular-pedestrian mixed flows in the evacuation zone. s.l., The 89th transportation annual meeting of the Transportation Research Board.

Zhang, X. \& Chang, G., 2011. Cellular automata-based model for simulating vehicular-pedestrian mixed flows in a congested network. Transportation Research Record: Journal of the Transportation Research Board, 2234(1), pp. 116-124. 